\title{
A simple model of biofilm growth in a porous medium that accounts for detachment and attachment of suspended biomass and their contribution to substrate degradation $\dagger$
}

\author{
HARRY J. GAEBLER and HERMANN J. EBERL \\ Department of Mathematics and Statistics, University of Guelph, \\ Guelph, Ontario, Canada N1G2W1 \\ email: gaeblerh@uoguelph.ca,heberl@uoguelph.ca
}

(Received 5 July 2017; revised 18 March 2018; accepted 19 March 2018; first published online 12 April 2018)

\begin{abstract}
We derive a macroscopic model for biofilm formation in a porous medium reactor to investigate the role of suspended bacteria on reactor performance. The starting point is the mesoscopic one-dimensional Wanner-Gujer biofilm model. The following processes are included: hydrodynamics and transport of substrate in the reactor, biofilm and suspended bacteria growth in the pore space, attachment of suspended cells to the biofilm, and detachment of biofilm cells. The mesoscopic equations are up-scaled from the biofilm scale to the reactor scale, yielding a stiff system of balance laws, which we study numerically. We find that suspended bacteria and attachment can have a significant effect on biofilm reactor performance.
\end{abstract}

Key words: Balance laws, biofilm, porous medium

MSC (2010): 35Q92, 68U20, 92D25

\section{Introduction}

Bacteria form a large domain of prokaryotic microorganisms that can be found throughout the natural world. A bacterial biofilm is a layered aggregate formed by bacteria that become attached to each other or a submersed surface. Due to their complex structure, biofilms can adapt to many different environments, including living tissue, indwelling medical devices, natural aquatic systems, and food processing plants [3,7,22]. A characteristic feature of bacterial biofilms is their ability to encase themselves in extracellular polymeric substances (EPS), which provide both mechanical protection and protection against antimicrobials $[1,3,10,22]$.

Biofilms are inherently multi-scale structures. Individual cells form the microscale $(\sim 1$ micron), biofilm colonies represent the mesoscale $(\sim 10-1,000$ microns $)$; the macroscale

$\dagger$ This study was financially supported through an Ontario Graduate Scholarship and an Arthur D. Latornell Graduate Scholarships awarded to HJG, and through an NSERC Discovery Grant (HJE). Computing resources were made available by an NSERC Research Tool and Infrastructure Grant awarded to HJE. 
$(>1 \mathrm{~cm})$ is the scale of biofilm reactors and environmental system where biofilms form. Inside the biofilm, physical, chemical, and biological processes occur on very different characteristic time-scales [26]. Processes related to the change in volume of the biofilm (growth, decay, detachment) are much slower $\left(10^{4}-10^{7}\right.$ seconds) than the processes related to substrate-mass balance (diffusion, convection, reaction), which occur on the order of $10^{-3}-10^{3}$ seconds $[26,34]$. A commonly adapted approach to deal with these time-scale discrepancies is to assume the system to be in quasi-steady state $[26,34]$. On the other hand, no common strategy for the spatial multiscale properties exists. In many instances, macroscale models are formulated for the reactor and mesoscale models are simply embedded in an ad hoc fashion. Such models, if they require a full numerical resolution of the mesoscale model can become computationally expensive, wherefore often simplified empirical or heuristic relationships are used to replace them, e.g. for substrate fluxes between the biofilm colonies and the aqueous phase in the reactor.

Biofilms often form in saturated porous media, such as soils, or certain technical reactors, such as sand filters of packed bed reactors, which prompts the need for the development of models for such an environment. Biofilms are often used in the environmental industry to contain spilled contaminants from entering nearby water sources through plugging the pore channels [31]. Other applications for subsurface biofilms include the following: subsurface remediation, oil recovery, and ground water protection [21,32]. In this case, biofilms sustain growth in the pore space of a porous medium. In these systems a growth substrate travels through the porous medium and biomass is produced through the consumption of such substrate. As the biofilm forms on the substratum, different factors influence its behaviour. One factor that affects the structure of the biofilm is detachment. In traditional models, whether they be continuously stirred tank reactors (CSTR) or plug flow reactors (PFR), mass can become detached from the surface due to a variety of different factors $[1,3,17,22]$.

Biofilm detachment is a complex process by which multiple mechanisms are involved. The detachment process mediates biofilm accumulation, affects long-term behaviour of the biofilm [3], and contributes to the production of suspended flocs [23], and downstream colonization [30]. Throughout the biofilm modelling literature several different detachment rates have been proposed and their effect on the formation of a biofilm has been investigated. Common mesoscopic detachment descriptions consist of a constant detachment rate, detachment rate proportional to biofilm thickness, and detachment rates that depend on hydrodynamic shear forces. It was found in [3] that the mesoscopic detachment expression may affect the biofilm's ability to establish itself and whether or not the biofilm will clog the channel. However, when mesoscopic models are upscaled to the reactor scale (macroscale) the mesoscopic detachment description may affect the macroscopic model. When the porous medium is far from clogging, the authors in [1] found that the mesoscopic detachment rate that depends on bulk flow hydrodynamics behaved quantitatively like the constant detachment rate and had an effect on the macroscopic reactor. However, the shear dependent detachment rate behaved like the proportional attachment rate and vanished in the macroscopic limit causing no effect on the macroscopic model. Under conditions where the porous medium was close to the clogging state, the bulk flow hydrodynamics detachment rate behaved more like the shear force detachment rate and caused the detachment rate to blow up [1]. The findings 
in [1] allow future models to simplify their detachment expression based on the reactor regime.

Another factor to consider is the contribution of suspended bacteria to both biofilm formation and consumption of substrate. The results in [22], in the case of a CSTR, indicate that suspended biomass is more efficient at substrate removal than attached biomass, as they are exposed to bulk flow substrate concentrations, whereas in biofilms bacteria in inner layers can experience substrate limitations. By considering suspended bacteria two issues arise [22]. First, the interaction between the biofilm and the suspended bacteria must be described. We model the interaction between the biofilm and the suspended bacteria by two generic functions that represent attachment and detachment processes. Second, the suspended biomass must be characterized. We describe the suspended bacteria similar to attached biomass and not individual planktonic cells, i.e. we implicitly assume that detached biomass is present in the form of small flocs. This characterization allows for suspended biomass to be modelled using the same growth parameters as biofilm biomass.

An important aspect of our study is to determine how incorporating a reattachment process at the mesoscale (the actual biofilm scale on the order of 100 micrometre to $\mathrm{mm}$ ) contributes to macroscale model (the scale of the reactor, on the order of $\mathrm{cm}$ to $\mathrm{m}$ ). Such attachment and reattachment of suspended biomass is considered an important aspect of the biofilm growth cycle [30]. Throughout the biofilm literature, biofilm attachment is not well understood [22]. Models where attachment of suspended bacteria is neglected can only simulate a reactor where a biofilm is already established, they cannot simulate and study potential formation of a biofilm inside a clean reactor. In many cases, attachment of suspended bacteria is included in the model $[4,17,22]$; however, attachment functions are primarily chosen out of mathematical convenience. In [17], the authors study a simple Freter model, where a fraction of cells are able to adhere to the surface; however, this fraction is a decreasing function of wall occupancy. In [22], a reactor scale CSTR model is formulated and attachment is assumed to occur at a constant rate, i.e. attachment is proportional to the amount of suspended bacteria. By first formulating a mesoscopic model with attachment, we can investigate whether or not attachment affects the macroscopic reactor.

To investigate the effects of mesoscopic processes on the macroscopic reactor, we upscale our porous medium biofilm model from the mesoscale to the macroscale. Upscaling techniques, in the form of volume/spatial averaging, have been used in a variety of studies over the last decade, with emphases ranging from biofilm growth, pore scale transport, biofilm performance, to $\mathrm{CO}_{2}$ sequestration in porous media $[1,9,15,18,36]$. By upscaling the biofilm model, characteristics of the mesoscale model can more accurately be incorporated in the macroscale model, rather than by directly formulating a macroscale model, as in [17,22]. Moreover, macroscopic model parameters can be naturally expressed in terms of the mesoscopic parameters [36]. Existing multiscale studies of biofilms tend to focus on the correct treatment of substrate transport at the expense of making simplifications to biofilm growth processes. An alternative is to start with a one-dimensional mesoscopic biofilm model and couple it with a macroscopic transport equation for the reactor, similar to [1].

A seminal one-dimensional mesoscopic biofilm model was introduced by [35] and has been the basis for most biofilm engineering applications over the past few decades [34]. The 
model consists of a mass balance based on conservation principles. Here, the underlying assumptions are that (i) the biofilm covers the substratum in a homogeneous layer, (ii) the biofilm is stratified, i.e. its properties change only in the direction perpendicular to the substratum, (iii) the biofilm is incompressible; thus, biomass production is translated $1: 1$ into expansion of the biofilm, and (iv) the flux of substrate into the biofilm follows Fick's first law [35]. These substrate fluxes are typically computed based on a steady-state assumption, based on time scale disparity. In the original model, specific assumptions of microbial growth were avoided, which allows the model to be adapted to any rate law or empirical formula that may be applicable to the problem at hand.

Our starting point is the traditional one-dimensional Wanner-Gujer biofilm model [35]. Mesoscopic processes included in the model are biomass production, substrate degradation, cell death, and biofilm detachment similar to [1,3,22]. Unlike the model described in [9] that assumes all interfaces are static resulting in biofilm growth translating to an increase in biomass density, but not biofilm thickness, we assume in accordance with traditional 1-D biofilm models that the biofilm density remains constant but thickness increases.

In a recent study that investigated the effect of mesoscopic detachment expressions on the macroscopic reactor [1], a major limiting assumption was made regarding suspended bacteria. It was assumed that after biomass becomes detached from the reactor wall, it is immediately washed out from the reactor. This assumption may be valid at most if the flow velocity is high enough and the reactor small enough. However, suspended bacteria, to some extent, are always present in biofilm reactors [22]. At lower flow velocities, biomass that becomes detached may have the ability to reattach to the substratum further down the flow channel. In this study, we introduce suspended bacteria and attachment of suspended bacteria to the biofilm and investigate the effects on the reactor performance.

Overall the objectives of this study are to (a) develop a model for the formation of a biofilm in a porous medium, (b) investigate the role of suspended bacteria, and (c) determine the effects of attachment of suspended bacteria to the biofilm layer on the solution of the system and on overall reactor performance.

The derivation of equations begins with a compartmentalizing process on the mesoscale. The macroscale model is then constructed by refining the mesoscale compartmental description. Underlying here is the assumption that the mesoscopic compartment is negligibly small compared to the macroscopic system size, wherefore the macroscale model is obtained by passing the mesoscopic compartment size to zero.

The resulting macroscale model is described by a non-linear convection-reaction equation in which the parameters of the macroscale model can be inferred from the parameters of the mesoscale model. Due to complexity of the system, limited knowledge may be obtained through analytical techniques. Here, we employ a numerical method for solving a system of non-linear hyperbolic partial differential equations and investigate the effects of parameters on solutions.

In [33], the starting point for the simulation of biofilm growth in a porous medium was similar to ours, in that in main flow direction a sub-division in mesoscopic compartments is carried out; however, the transition to the continuous limit was not made. Instead, in [33], a simulation of the growth process consisted of the simulation of a small finite number of such mesoscopic compartments. 


\section{Mathematical model}

\subsection{Model assumptions}

We consider biofilm growth in a homogeneous porous medium with a well-defined primary flow direction. A growth promoting substrate and suspended bacteria are transported in the pore space by convection and substrate is consumed to promote biomass growth both on the substratum and in the bulk liquid. As the biofilm changes thickness, biomass can become detached from the biofilm and enter the aqueous phase; vice versa, biomass from the aqueous phase is able to (re-)attach to the biofilm. We assume that the flow rate through the porous medium is kept constant.

To derive a mathematical model for the biofilm growth process on the macroscale, the following mesoscopic assumptions are made:

(1) The porous medium can be described by parallel, non-communicating flow channels of width $\epsilon[L][16]$. The flow in these channel is driven by specifying the reactor flow rate.

(2) In the flow direction, the flow channels are compartmentalized by breaking them down into small segments of length $\epsilon[L]$ each, cf. Figure 1.

(3) We assume, for simplicity, that the porous material is homogeneous, i.e. the pore space is the same for each compartment of size $\epsilon \times \epsilon$.

(4) In each compartment, biofilm can form in the pore space, where the walls of the flow channel form the substratum. Biofilm growth is described by a 1-D biofilm model, i.e. we assume that in each compartment of size $\epsilon \times \epsilon$ the biofilm is homogeneous. We use the Wanner-Gujer 1-D biofilm model, i.e. assume that the biomass density in the biofilm is constant and that production of new biomass is $1: 1$ translated into biofilm expansion $[3,22,34]$. Similarly, biomass loss is equivalent to a corresponding biofilm shrinkage.

(5) In addition to biofilm that is attached to the porous material, we account for suspended bacteria in the aqueous phase, which covers the area of the pore space not occupied by biofilm. In each compartment of size $\epsilon \times \epsilon$, it is assumed that the suspended biomass is completely mixed in the aqueous phase and that the volume occupied by suspended biomass is negligible, i.e. suspended biomass is dissolved in accordance with $[8,12,22]$. Transfer of biomass between the biofilm and the aqueous phase takes place as a consequence of detachment and attachment.

(6) We assume that attachment process of suspended biomass to the substratum is the same process as the attachment of suspended biomass to an existing biofilm colony, i.e. we do not consider properties of the biofilm when describing the attachment process.

(7) A non-reproducing growth limiting substrate is added to the reactor at the upstream boundary. In the aqueous phase, we assume the growth limiting substrate to be completely mixed. In the biofilm, the substrate diffuses from the biofilm/water interface into the inner layers, i.e. perpendicular to the main flow direction of the reactor. In the biofilm phase, the substrate is utilized by biofilm bacteria for growth; in the aqueous phase, utilized by suspended bacteria. 


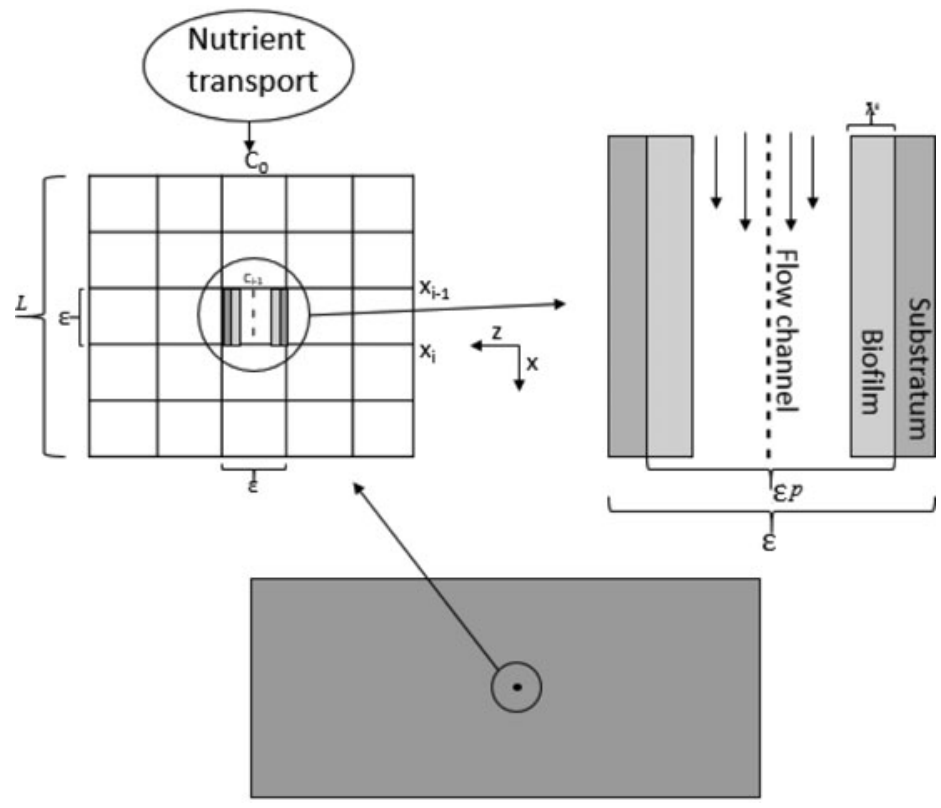

FiguRE 1. Representation of the macroscopic reactor (bottom) with macroscopic compartmentalization (top left) and mesoscopic cells of size $\epsilon \times \epsilon$ (top right). Each cell contains a pore void fraction $p$ and biofilm $\lambda^{\epsilon}$ forms on both of the boundary walls. To obtain the macroscopic description, $\epsilon$ is passed to the continuous limit, $\epsilon \rightarrow 0$, i.e. the mesoscopic cell is reduced to a point.

(8) The flow rate in each channel of width $\epsilon$ is the same and remains constant. Biofilm growth narrows the flow path, i.e. under the assumption of a constant flow rate, can lead to faster local flow velocities. Growth limiting substrate and suspended biomass are transported by convection in the flow channels.

The macroscopic model is obtained from the mesoscopic model by refining the compartments and taking $\epsilon$ to the continuous limit, $\epsilon \rightarrow 0$. A representation of both the macroscopic and mesoscopic model can be seen in Figure 1.

\subsection{Governing equations}

2.2.1 Substrate concentration in the biofilm and substrate flux between aqueous phase and biofilm

Substrate inside the biofilm is transported by Fickian diffusion in the $z$-direction, perpendicular to the main flow direction of the reactor, and is consumed by bacteria for growth. We invoke the standard time-scale argument from biofilm modelling that substrate diffusion and utilization are much faster than biofilm growth, which allows a quasi-steady-state assumption for the substrate [34]. If $\lambda^{\epsilon}[L]$ is the thickness of the biofilm, then the substrate concentration $c(z)\left[M \cdot L^{-2}\right]$ in the biofilm is given by the solution of the two-point boundary value problem

$$
D \frac{d^{2} c}{d z^{2}}=\frac{X_{\infty}}{Y_{\lambda}} f(c), \quad \frac{d c}{d z}(0)=0, \quad c\left(\lambda^{\epsilon}\right)=C, \quad 0<z<\lambda^{\epsilon},
$$


where $D\left[L^{2} \cdot T^{-1}\right]$ is the diffusion coefficient of the substrate in the biofilm, $X_{\infty}$ $\left[M \cdot L^{-2}\right]$ is the biomass density in the biofilm, and $C\left[M \cdot L^{-2}\right]$ is the substrate concentration in the completely mixed aqueous phase. In the quasi-steady model (2.1), the bulk concentration $\mathrm{C}$ in the aqueous phase is treated as a constant; however, it is time dependent on the reactor scale. Here, all masses are expressed in gCOD equivalents, where gCOD refers to the amount of oxygen required for the chemical reaction to take place, as is common in biofilm modelling [28]. $Y_{\lambda}[-]$ is the yield coefficient that indicates the amount of biomass produced from a mass unit of substrate, e.g. expressed

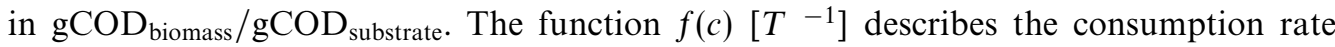
of substrate by biomass inside the biofilm layer. This could be the first-order or Monod kinetics, i.e.

$$
f_{L}(c)=\frac{\mu_{\lambda}}{\kappa_{\lambda}} c \quad \text { or } \quad f_{M}(c)=\frac{\mu_{\lambda} c}{\kappa_{\lambda}+c},
$$

where $\mu_{\lambda}\left[T^{-1}\right]$ is the per capita growth rate for the biofilm. In the case of Monod kinetics, $\kappa_{\lambda}\left[M \cdot L^{-2}\right]$ is the concentration at which the biofilm attains half-maximal growth. The first-order kinetics is a special case of Monod kinetics for $C \ll \kappa_{\lambda}$. The flux $J^{\epsilon}\left[M \cdot L^{-1} \cdot T^{-1}\right]$ of substrate into the biofilm is computed from $c(z)$ as

$$
J^{\epsilon}\left(C, \lambda^{\epsilon}\right):=\left.D \frac{d c}{d z}\right|_{\lambda^{\epsilon}}
$$

\subsubsection{Mesoscopic biofilm model in the pore space}

The biofilm thickness in the $i$ th $\epsilon$-cell $\lambda_{i}^{\epsilon}$ is determined from a mass balance for biofilm growth, detachment, and re-attachment of bacteria. In our initial model formulation, we use a generic detachment expression $\hat{d}\left[T^{-1}\right]$ and a generic attachment expression $\tilde{a}$ $\left[L \cdot T^{-1}\right]$ for the $i$ th $\epsilon$-cell, both of which we make more precise later.

Our basic assumption is that across the biofilm, the biomass density is constant at $X_{\infty}$ and that newly produced biomass leads directly to an expansion of the one-dimensional biofilm with velocity $v\left[L \cdot T^{-1}\right]$. Following [1,22], this biomass velocity $v$ at location $z$ in the biofilm is obtained as the integral of the biomass production rate

$$
v(z, t)=\int_{0}^{z}\left(f\left(c_{i}(\xi)\right)-k_{d}\right) d \xi,
$$

where $k_{d}\left[T^{-1}\right]$ is the biomass death rate and $c_{i}=c_{i}(z)$ is the substrate concentration in the $i$ th $\epsilon$-cell at location $z$ in the biofilm according to (2.1). The time-dependency of $v$ stems from the fact that the substrate concentration in the aqueous phase, $C=C(t)$, changes with time. It enters (2.1) via the boundary condition, whence the integrand is time-dependent as well.

Thus, the one-dimensional biofilm thickness in the $i$ th cell evolves according to

$$
\frac{d \lambda_{i}^{\epsilon}}{d t}=v\left(\lambda^{\epsilon}(t), t\right)-\hat{d} \lambda_{i}^{\epsilon}+\tilde{a}
$$


or

$$
\frac{d \lambda_{i}^{\epsilon}}{d t}=\int_{0}^{\lambda_{i}^{\epsilon}} f\left(c_{i}\right) d z-k_{d} \lambda_{i}^{\epsilon}-\hat{d} \lambda_{i}^{\epsilon}+\tilde{a} .
$$

Here, it is important to note that we consider the detachment process to take place at the biofilm/aqueous interface only, as is common in 1-D biofilm modelling, see [34], but has also been applied in 2D models, e.g. [11], rather than using a volumetric detachment rate as in [24]. Underlying here is the notion that there is no detachment from the inner layers of the biofilm.

Integrating (2.1) once and using (2.3), we can write the integral on the right hand side in terms of the substrate flux as

$$
\int_{0}^{\lambda_{i}^{\epsilon}} f\left(c_{i}(z)\right) d z=\left.\frac{Y_{\lambda}}{X_{\infty}} D \frac{d c_{i}}{d z}\right|_{\lambda_{i}^{\epsilon}}=\frac{Y_{\lambda}}{X_{\infty}} J_{i}^{\epsilon}\left(C_{i}, \lambda_{i}^{\epsilon}\right) .
$$

In order to write all balance equations in terms of COD equivalents, we convert thickness to bacterial mass (expressed in terms of gCOD equivalents), by multiplying by the relative biofilm density $X_{\infty}$ and the length of the compartment $\epsilon$. Using $\eta:=Y_{\lambda} / X_{\infty}$, equation (2.5) can be rewritten as

$$
\epsilon X_{\infty} \frac{d \lambda_{i}^{\epsilon}}{d t}=\epsilon X_{\infty} \eta J_{i}^{\epsilon}\left(C_{i}, \lambda_{i}^{\epsilon}\right)-\epsilon X_{\infty} k_{d} \lambda_{i}^{\epsilon}-\epsilon X_{\infty} \hat{d} \lambda_{i}^{\epsilon}+\epsilon X_{\infty} \tilde{a}
$$

The substrate concentration in the aqueous phase is given by

$$
\frac{d\left(V_{i} C_{i}\right)}{d t}=\epsilon Q C_{i-1}-\epsilon Q C_{i}-2 \epsilon J_{i}^{\epsilon}\left(C_{i}, \lambda_{i}^{\epsilon}\right)-\frac{1}{Y_{u}} g\left(C_{i}\right) U_{i} V_{i}
$$

where $\epsilon Q[\mathrm{~L}]\left[\mathrm{L} \cdot \mathrm{T}^{-1}\right]$ is the flow rate in a channel of thickness $\epsilon$. The flow rate in each channel is obtained under the assumption that the global flow rate $Q$ through the entire reactor of width $H$ [L] remains constant and is divided evenly across each channel of width $\epsilon$. In (2.7), $V_{i}\left[L^{2}\right]$ is the volume of the aqueous phase, which is a function of compartmental size $\epsilon$ and biofilm thickness $\lambda_{i}^{\epsilon}$, namely

$$
V_{i}=\epsilon\left(\epsilon p-2 \lambda_{i}^{\epsilon}\right)
$$

where $p[-]$ is the void fraction of the porous medium, see also Figure 1. The first two terms on the right hand side of (2.7) describe the substrate inflow from the upstream compartment with concentration $C_{i-1}$ and outflow into the downstream compartment with concentration $C_{i}$, respectively. The third term on the right hand side of (2.7) describes the flux of substrate into the biofilm, which also depends on $\epsilon$. The fourth term on the right hand side of (2.7) models the loss of substrate concentration due to reproduction of suspended biomass in the aqueous phase, where the function $g\left(C_{i}\right)\left[T^{-1}\right]$ is a generic bacterial growth rate function for the $i$ th compartment; $U_{i}\left[M \cdot L^{-2}\right]$ denotes the concentration of suspended biomass in the aqueous phase. As in the case of bacterial growth in biofilms, we propose suspended bacteria growth $g(C)$ to be modelled by the 
first-order or Monod kinetics, i.e.

$$
g(C)=g_{L}(C):=\frac{\mu_{u} C}{\kappa_{u}} \quad \text { or } \quad g(C)=g_{M}(C)=\frac{\mu_{u} C}{\kappa_{u}+C}
$$

where $\mu_{u}\left[T^{-1}\right]$ is the per capita growth rate, and $\kappa_{u}\left[M \cdot L^{-2}\right]$ is the concentration at which growth is half-maximal.

Finally, the mesoscopic model is completed by constructing a mass balance that describes the concentration of suspended bacteria. We have

$$
\frac{d\left(V_{i} U_{i}\right)}{d t}=\epsilon Q U_{i-1}-\epsilon Q U_{i}+2 \epsilon X_{\infty} \hat{d} \lambda_{i}^{\epsilon}-2 \hat{a} V_{i} U_{i}+g\left(C_{i}\right) U_{i} V_{i}
$$

where the first two terms on the right hand side of (2.10) describe the inflow of biomass from the upstream compartment with concentration $U_{i-1}$ and the outflow into the downstream compartment with concentration $U_{i}$. The third term on the right hand side accounts for detachment from the biofilm and the fourth term for attachment to the biofilm, where $\hat{a}\left[T^{-1}\right]$ is the attachment rate. The attachment terms in (2.10) and in (2.6) must be equal, wherefore they are related by $\tilde{a}=\hat{a} U V /\left(\epsilon X_{\infty}\right)$. Finally, the fifth term describes growth of suspended biomass due to substrate concentration.

Using (2.8) along with the mesoscopic equations (2.6), (2.7), (2.10), we obtain

$$
\begin{aligned}
\epsilon \frac{d\left(\left[\epsilon p-2 \lambda_{i}^{\epsilon}\right] C_{i}\right)}{d t}= & \epsilon Q\left(C_{i-1}-C_{i}\right)-2 \epsilon J_{i}^{\epsilon}\left(C_{i}, \lambda_{i}^{\epsilon}\right)-\frac{\epsilon}{Y_{u}} g\left(C_{i}\right) U_{i}\left[\epsilon p-2 \lambda_{i}^{\epsilon}\right], \\
\epsilon \frac{d\left(\left[\epsilon p-2 \lambda_{i}^{\epsilon}\right] U_{i}\right)}{d t}= & \epsilon Q\left(U_{i-1}-U_{i}\right)+2 \epsilon X_{\infty} \hat{d} \lambda_{i}^{\epsilon}-2 \hat{a} \epsilon\left[\epsilon p-2 \lambda_{i}^{\epsilon}\right] U_{i} \\
& +\epsilon g\left(C_{i}\right) U_{i}\left[\epsilon p-2 \lambda_{i}^{\epsilon}\right], \\
\epsilon X_{\infty} \frac{d \lambda_{i}^{\epsilon}}{d t}= & \epsilon X_{\infty} \eta J_{i}^{\epsilon}\left(C_{i}, \lambda_{i}^{\epsilon}\right)-\epsilon X_{\infty} k_{d} \lambda_{i}^{\epsilon}-\epsilon X_{\infty} \hat{d} \lambda_{i}^{\epsilon} \\
& +\hat{a} \epsilon\left[\epsilon p-2 \lambda_{i}^{\epsilon}\right] U_{i} .
\end{aligned}
$$

We non-dimensionalize the biofilm thickness $\lambda_{i}^{\epsilon}$ with respect to the compartmental size $\epsilon$. Then, $\lambda_{i}[-]$ becomes the fraction of the pore space that is occupied by a biofilm growing on one of the boundary walls. The non-dimensional variable is given by

$$
\lambda_{i}:=\frac{\lambda_{i}^{\epsilon}}{\epsilon}
$$

where $\lambda_{i}$ must satisfy

$$
0 \leqslant \lambda_{i}<\frac{p}{2}
$$

If the second inequality is violated the model breaks down due to clogging of the flow 
channel. Substituting (2.14) into (2.11)-(2.13) finally yields the mesoscopic system:

$$
\begin{aligned}
\frac{d\left(\left[p-2 \lambda_{i}\right] C_{i}\right)}{d t}= & Q\left(\frac{C_{i-1}-C_{i}}{\epsilon}\right)-2 \frac{J_{i}^{\epsilon}\left(C_{i}, \lambda_{i}^{\epsilon}\right)}{\epsilon}-\frac{\left[p-2 \lambda_{i}\right]}{Y_{u}} g\left(C_{i}\right) U_{i}, \\
\frac{d\left(\left[p-2 \lambda_{i}\right] U_{i}\right)}{d t}= & Q\left(\frac{U_{i-1}-U_{i}}{\epsilon}\right)+2 X_{\infty} \hat{d} \lambda_{i}-2 \hat{a}\left[p-2 \lambda_{i}\right] U_{i} \\
& +g\left(C_{i}\right)\left[p-2 \lambda_{i}\right] U_{i}, \\
\frac{d \lambda_{i}}{d t}= & \eta \frac{J_{i}^{\epsilon}\left(C_{i}, \lambda_{i}^{\epsilon}\right)}{\epsilon}-k_{d} \lambda_{i}-\hat{d} \lambda_{i}+\frac{\hat{a}}{X_{\infty}}\left[p-2 \lambda_{i}\right] U_{i} .
\end{aligned}
$$

\subsubsection{Macroscopic biofilm model}

The macroscopic model is derived from the mesoscopic compartment model (2.16), (2.17), and (2.18) by passing $\epsilon \rightarrow 0$, i.e. making the discrete mesoscopic compartments smaller and smaller, until their spatial extension is negligible on the reactor scale, cf. also Figure 1. In preparation of this, we first investigate how the substrate flux into the biofilm depends on $\epsilon$ as $\epsilon \rightarrow 0$, for the first-order and Monod kinetics.

Proposition 2.1 Let $c(z)$ be the solution to the two point boundary value problem

$$
\frac{d^{2} c}{d z^{2}}=\frac{\mu_{\lambda}}{\kappa_{\lambda}} c, \quad \frac{d c}{d z}(0)=0, \quad c\left(\lambda^{\epsilon}\right)=C, \quad 0<z<\lambda^{\epsilon} .
$$

Then the flux of substrate into the biofilm is such that

$$
J^{\epsilon}\left(C, \lambda_{i}^{\epsilon}\right)=D \frac{d c}{d z}\left(\lambda^{\epsilon}\right)=\mathcal{O}(\epsilon)
$$

Proof Let $k:=\mu_{\lambda} / \kappa_{\lambda}$, then the solution of the boundary value problem is

$$
c(z)=C \frac{e^{-\sqrt{k} z}+e^{\sqrt{k} z}}{e^{-\sqrt{k} \lambda^{\epsilon}}+e^{\sqrt{k} \lambda^{\epsilon}}} .
$$

Hence, by differentiation, the flux into the biofilm is given by

$$
J^{\epsilon}\left(C, \lambda^{\epsilon}\right)=\left.D \frac{d c}{d z}\right|_{\lambda^{\epsilon}}=D C \sqrt{k} \tanh \left(\sqrt{k} \lambda^{\epsilon}\right)
$$

Using the variable transformation (2.14) and taking the limit as $\epsilon \rightarrow 0$, we have

$$
\lim _{\epsilon \rightarrow 0} \frac{J(C, \lambda \epsilon)}{\epsilon}=\lim _{\epsilon \rightarrow 0} \frac{D C \sqrt{k} \tanh (\epsilon \sqrt{k} \lambda)}{\epsilon}=D C k \lambda=\text { const }
$$

hence, $J^{\epsilon}\left(C, \lambda^{\epsilon}\right)=\mathcal{O}(\epsilon)$.

Proposition 2.2 Let $c(z)$ be the solution to the two point boundary value problem

$$
\frac{d^{2} c}{d z^{2}}=\frac{\mu_{\lambda} c}{\kappa_{\lambda}+c}, \quad \frac{d c}{d z}(0)=0, \quad c\left(\lambda^{\epsilon}\right)=C, \quad 0<z<\lambda^{\epsilon} .
$$


Then, the flux of substrate into the biofilm is such that

$$
J^{\epsilon}\left(C, \lambda^{\epsilon}\right)=D \frac{d c}{d z}\left(\lambda^{\epsilon}\right)=\mathcal{O}(\epsilon)
$$

Proof Note that $c=0$ is a lower solution for (2.19) and $c=C$ is an upper solution. Therefore, $0 \leqslant c(z) \leqslant C$ from standard comparison theorems for two-point boundary value problems [6].

We consider now the following auxiliary linear boundary value problems

$$
\frac{d^{2} c_{1}}{d z^{2}}=\frac{\mu_{\lambda} c_{1}}{\kappa_{\lambda}+C}, \quad \frac{d c_{1}}{d z}(0)=0, \quad c_{1}\left(\lambda^{\epsilon}\right)=C,
$$

and

$$
\frac{d^{2} c_{2}}{d z^{2}}=\frac{\mu_{\lambda} C}{\kappa_{\lambda}+C}, \quad \frac{d c_{2}}{d z}(0)=0, \quad c_{2}\left(\lambda^{\epsilon}\right)=C .
$$

From

$$
\frac{\mu_{\lambda} c}{\kappa_{\lambda}+C} \leqslant \frac{\mu_{\lambda} c}{\kappa_{\lambda}+c} \leqslant \frac{\mu_{\lambda} C}{\kappa_{\lambda}+C}
$$

the comparison theorems in [6] imply

$$
c_{2}(z) \leqslant c(z) \leqslant c_{1}(z)
$$

The upper estimate $c_{1}(z)$ is analogous to Proposition 2.1, but with $k:=\mu_{\lambda} /\left(\kappa_{\lambda}+C\right)$. Thus,

$$
J_{1}^{\epsilon}\left(C, \lambda_{i}^{\epsilon}\right)=D \frac{d c_{1}}{d z}\left(\lambda^{\epsilon}\right)=D C \sqrt{\frac{\mu_{\lambda}}{\kappa_{\lambda}+C}} \tanh \left(\frac{\mu_{\lambda}}{\kappa_{\lambda}+C} \lambda^{\epsilon}\right),
$$

and, therefore,

$$
\lim _{\epsilon \rightarrow 0} \frac{J_{1}(C, \lambda \epsilon)}{\epsilon}=\lim _{\epsilon \rightarrow 0} \frac{D C \sqrt{\frac{\mu_{\lambda}}{\kappa_{\lambda}+C}} \tanh \left(\epsilon \sqrt{\frac{\mu_{\lambda}}{\kappa_{\lambda}+C}} \lambda\right)}{\epsilon}=D C \frac{\mu_{\lambda}}{\kappa_{\lambda}+C} \lambda=\text { const. }
$$

Hence, $J_{1}^{\epsilon}\left(C, \lambda^{\epsilon}\right)=\mathcal{O}(\epsilon)$, as above in Proposition 2.1. The lower estimate $c_{2}(z)$ is

$$
c_{2}(z)=\frac{1}{2} \frac{\mu_{\lambda} C}{\kappa_{\lambda}+C}\left(z^{2}-\lambda^{\epsilon 2}\right)+C
$$

thus by differentiation we obtain

$$
J_{2}^{\epsilon}\left(C, \lambda_{i}^{\epsilon}\right)=D \frac{d c_{2}}{d z}\left(\lambda^{\epsilon}\right)=\frac{D \mu_{\lambda} C \lambda^{\epsilon}}{\kappa_{\lambda}+C} .
$$

Using the variable transformation (2.14) and taking the limit as $\epsilon \rightarrow 0$, we have 


$$
\lim _{\epsilon \rightarrow 0} \frac{J_{2}(C, \lambda \epsilon)}{\epsilon}=\lim _{\epsilon \rightarrow 0} \frac{D \frac{\mu_{\lambda} C}{\kappa_{\lambda}+C} \epsilon \lambda}{\epsilon}=D \frac{\mu_{\lambda} C}{\kappa_{\lambda}+C} \lambda=\text { const. }
$$

Thus, also $J_{2}^{\epsilon}\left(C, \lambda^{\epsilon}\right)=\mathcal{O}(\epsilon)$.

Moreover, since $c_{1}\left(\lambda^{\epsilon}\right)=c\left(\lambda^{\epsilon}\right)=c_{2}\left(\lambda^{\epsilon}\right)=C$ it follows from (2.20) that

$$
\frac{d c_{2}}{d z}\left(\lambda^{\epsilon}\right) \geqslant \frac{d c}{d z}\left(\lambda^{\epsilon}\right) \geqslant \frac{d c_{1}}{d z}\left(\lambda^{\epsilon}\right) .
$$

Therefore, for small enough $\epsilon$, we find that $d c / d z\left(\lambda^{\epsilon}\right)$ is bounded from below and above by two $\mathcal{O}(\epsilon)$ functions. By the squeeze theorem, we have, therefore, with the relationship between substrate flux and gradient (2.3)

$$
J^{\epsilon}\left(C, \lambda^{\epsilon}\right)=\mathcal{O}(\epsilon)
$$

Remark 2.3 In the above proof, we used that the fluxes of the super- and sub-solution on the Monod boundary value problem can be estimated from above and below by evaluations of the Monod equation. In [2] and [19], using very different approaches, such terms were derived as an approximation of the flux itself under the assumption that the biofilm is thin. In the simulations of [1], this algebraic approximation of the flux was used instead of computing it from the two-point boundary value problem (2.1), a simplification that we remove below.

Similarly, for the upscaling of the mesoscopic model to the macroscale, the behaviour of the detachment and attachment rates $\hat{d}$ and $\hat{a}$ as $\epsilon \rightarrow 0$ will be important. Various detachment rates have been used in the 1-D biofilm modelling literature [3, 22, 27, 29]; on the other hand very little is known about attachment rate functions, which often are chosen primarily out of mathematical convenience $[4,17,22]$. To account for that, we formulate our result for generic expressions and choose specific representatives below.

Proposition 2.4 Consider (2.16)-(2.18) with generic detachment rate $\hat{d}=\mathcal{O}\left(\epsilon^{n_{1}}\right)$ and attachment rate $\hat{a}=\mathcal{O}\left(\epsilon^{n_{2}}\right)$ with $n_{1}, n_{2} \geqslant 0$. Assume the flux into the biofilm is such that $J^{\epsilon}\left(C, \lambda^{\epsilon}\right)=\mathcal{O}(\epsilon)$. By passing $\epsilon \rightarrow 0$ to the continuous limit the macroscopic limit of the system becomes

$$
\frac{\partial}{\partial t}\left(\begin{array}{c}
(p-2 \lambda) C \\
(p-2 \lambda) U \\
\lambda
\end{array}\right)+\frac{\partial}{\partial x}\left(\begin{array}{c}
Q C \\
Q U \\
0
\end{array}\right)=\left(\begin{array}{c}
-2 J(C, \lambda)-\frac{[p-2 \lambda]}{Y_{u}} g(C) U \\
2 X_{\infty} d \lambda-2 a[p-2 \lambda] U+g(C)[p-2 \lambda] U \\
\eta J(C, \lambda)-k_{d} \lambda-d \lambda+\frac{a}{X_{\infty}}[p-2 \lambda] U
\end{array}\right)
$$

where $J(C, \lambda):=\lim _{\epsilon \rightarrow 0} \frac{J_{i}^{\epsilon}\left(C, \lambda_{i}^{\epsilon}\right)}{\epsilon}, d:=\lim _{\epsilon \rightarrow 0} \hat{d}$, and $a:=\lim _{\epsilon \rightarrow 0} \hat{a}$.

Proof By passing to the continuous limit as $\epsilon \rightarrow 0$, we have from (2.16) that

$$
\lim _{\epsilon \rightarrow 0} \frac{C_{i}-C_{i-1}}{\epsilon}=\frac{\partial C}{\partial x}
$$


and similar for $U_{i}$. By assumption on $J^{\epsilon}\left(C, \lambda^{\epsilon}\right), \hat{d}$ and $\hat{a}$, the resulting macroscopic model is given by (2.21).

Remark 2.5 Per Propositions 2.1 and 2.2, both linear and Monod kinetics satisfy the flux assumption in Proposition 2.4.

Remark 2.6 Proposition 2.4 is formulated for a generic detachment rate expression. Several specific detachment expressions have been used in the biofilm literature, some of which have been compiled in [1]. In [1], based on a review of earlier published work, four different mesoscopic detachment expressions, as a function of mesoscopic biofilm thickness $\tilde{\lambda}[L]$, were considered and their effect on the macroscopic model via volume averaging was investigated. The mesoscopic detachment expressions are

$$
\begin{aligned}
& \hat{d}_{1}(\tilde{\lambda})=\delta, \\
& \hat{d}_{2}(\tilde{\lambda})=\delta \tilde{\lambda}, \\
& \hat{d}_{3}(\tilde{\lambda})=\delta\left(\frac{\tau}{\tau_{0}}\right)^{v}, \quad 0<v<1, \\
& \hat{d}_{4}(\tilde{\lambda})=\delta \tau \tilde{\lambda},
\end{aligned}
$$

where in each case $\delta$ denotes a constant detachment rate coefficient, the dimension of which may be different in each case. $\tau=\tau(\tilde{\lambda})$ denotes the hydrodynamic shear rate (cf. [1] for its derivation and definition), $\tau_{0}$ is a reference shear rate, and the exponent $v$ was derived from experimental observations. In [1], it was found that detachment effects from $\hat{d}_{2}$ were negligible in the macroscopic limit; however, for $\hat{d}_{3}$ and $\hat{d}_{4}$, the effects of local hydrodynamic conditions carried over to the macroscale. Furthermore, it was found that for small $\tilde{\lambda} \ll \epsilon p / 2$, the model with $\hat{d}_{3}$ behaved similar to $\hat{d}_{1}$ and $\hat{d}_{4}$ behaved like $\hat{d}_{2}$. As $\tilde{\lambda} \rightarrow \epsilon p / 2$ the two detachment expressions that were depended on hydrodynamic conditions, $\hat{d}_{3}$ and $\hat{d}_{4}$, behaved similar, i.e the detachment rates blow up. In this paper, we investigate our model when $\lambda \ll \epsilon p / 2$, and therefore select $\hat{d}_{1}$ as our primary detachment rate expression, which could also be re-interpreted as $\hat{d}_{3}$. Then, we have $\hat{d} \epsilon=\mathcal{O}(\epsilon)$, i.e. $d$ in $(2.21)$ is a constant.

To complete the macroscopic model initial conditions on $C, U$, and $\lambda$ must be specified,

$$
\lambda(0, x)=\lambda_{0}(x), \quad C(0, x)=C_{0}(x), \quad U(0, x)=U_{0}(x)
$$

as well as boundary conditions for substrate and suspended biomass on inflow

$$
C(t, 0)=C_{0}(t), \quad U(t, 0)=U_{0}(t)
$$

The specific choice of these functions will be stated below where simulation studies are described. 


\subsection{Numerical treatment}

We discuss here briefly the numerical treatment for Monod growth kinetics for both biofilm and suspended bacteria, as this is what we will use in the simulations below. For more details on the numerical realization and convergence of the implemented method, see [14].

A variable transformation is made first in order to write the system (2.21) in the standard evolution equation form of balance laws. We introduce the new dependent variables:

$$
S:=(p-2 \lambda) C, \quad W:=(p-2 \lambda) U
$$

Then, the system (2.21), along with the Monod growth kinetics for suspended bacteria $g_{M}(C)$ given in (2.9), is obtained as

$$
\frac{\partial}{\partial t}\left(\begin{array}{c}
S \\
W \\
\lambda
\end{array}\right)+\frac{\partial}{\partial x}\left(\begin{array}{c}
\frac{Q S}{p-2 \lambda} \\
\frac{Q W}{p-2 \lambda} \\
0
\end{array}\right)=\left(\begin{array}{c}
-2 J\left(\frac{S}{p-2 \lambda}, \lambda\right)-\frac{1}{Y_{u}} \frac{\mu_{u} S W}{\kappa_{u}(p-2 \lambda)+S} \\
2 X_{\infty} d \lambda-2 a W+\frac{\mu_{u} S W}{k_{u}(p-2 \lambda)+S} \\
\eta J\left(\frac{S}{p-2 \lambda}, \lambda\right)-\left(k_{d}+d\right) \lambda+\frac{a W}{X_{\infty}}
\end{array}\right) .
$$

To solve (2.25), we use the Uniformly accurate Central Scheme of Order 2 (UCS2) proposed in [20]. See Appendix A for a description of the method. UCS2 is an implicit method that calculates two predictor (intermediate) steps, which are then used to calculate grid points at the next time step. Since the method is an implicit method, stiffness issues related to the varying characteristic timescales are avoided. This requires to solve nonlinear systems of three equations for three unknowns in each grid point. We use Newton's method for this task. To evaluate the fluxes, the two-point boundary value problem (2.1) is solved using a single shooting method based on interval bracketing and the Runge-KuttaFehlberg method RKF4(5) [5,13]. This requires the derivatives of the fluxes $J$, for which we do not have an analytic expression. In our implementation they are approximated by finite differencing.

The method was implemented in $\mathrm{C}$ and compiled and tested using GNU and Intel compilers (gcc version 5.0.0, icc version 17.0.2). Simulations were carried out on a standard Linux desktop workstation under Ubuntu 16.04. All plots were generated using MATLAB v. 8.6.0.267246 (R2015b).

In the case of first-order kinetics, which we do not pursue here further, the numerics simplifies substantially because the two-point boundary problem for the substrate can be solved analytically and exact expressions for the fluxes are available.

\section{Simulation results}

In the following sections, we provide simulation results of the system (2.24). Parameter values for all simulations are given in Table 1 and simulation configurations are summarized in Table 2 .

\subsection{A typical simulation}

To illustrate the model behaviour, two representative simulations are carried out: in the first one, the effect of suspended bacteria and attachment is neglected, i.e. the original 
Table 1. Model parameter values used in simulations

\begin{tabular}{lcccc}
\hline \hline Parameter & Symbol & Value & Unit & Reference \\
\hline Substrate inflow concentration & $C_{0}$ & 1.0 & $\mathrm{gm}^{-2}$ & Assumed \\
Suspended bacteria inflow concentration & $U_{0}$ & 0.0 & $\mathrm{gm}^{-2}$ & Assumed \\
Relative biofilm thickness at inflow & $\lambda_{0}$ & 0.0025 & $\ldots$ & Assumed \\
Biomass density & $X_{\infty}$ & 100.0 & $\mathrm{gm}^{-2}$ & {$[34]$} \\
Biofilm maximum growth rate & $\mu_{\lambda}$ & 6.0 & $\mathrm{~d}^{-1}$ & {$[34]$} \\
Biofilm half saturation constant & $\kappa_{\lambda}$ & 4.0 & $\mathrm{gm}^{-2}$ & {$[34]$} \\
Biofilm yield coefficient & $Y_{\lambda}$ & 0.63 & $\ldots$ & {$[34]$} \\
Suspended bacteria maximum growth rate & $\mu_{u}$ & 6.0 & $\mathrm{~d}^{-1}$ & {$[34]$} \\
Suspended bacteria half saturation constant & $\kappa_{u}$ & 4.0 & $\mathrm{gm}^{-2}$ & {$[34]$} \\
Suspended bacteria yield coefficient & $Y_{u}$ & 0.63 & $\ldots$ & {$[34]$} \\
Void fraction & $p$ & 0.5 & $\ldots$ & Assumed \\
Biofilm natural cell death rate & $k_{d}$ & 0.4 & $\mathrm{~d}^{-1}$ & {$[34]$} \\
Detachment coefficient & $d$ & 0.5 & $\mathrm{~d}^{-1}$ & {$[1]$} \\
Attachment coefficient & $a$ & Varied & $\mathrm{d}^{-1}$ & Assumed \\
Flow velocity & $Q$ & 0.05 & $\mathrm{md}^{-1}$ & Assumed \\
Diffusion coefficient & $D$ & $10^{-4}$ & $\mathrm{~m}^{2} \mathrm{~d}^{-1}$ & {$[34]$} \\
Reactor length & $L$ & 0.15 & $\mathrm{~m}^{-1}$ & Assumed \\
\hline \hline
\end{tabular}

model of [1] is solved; in the second one, they are considered, i.e. the new model (2.21) is solved.

In these simulations, the initial conditions for substrate concentration, suspended bacteria concentration, and relative biofilm thickness are set to constants, $C_{0}=1.0\left[\mathrm{gm}^{-2}\right]$, $U_{0}=0.0\left[\mathrm{gm}^{-2}\right]$, and $\lambda_{0}=0.0025[-]$, respectively. Along the boundary, these same values are used for the substrate concentration and the suspended bacteria concentration. To obtain the relative biofilm thickness along the boundary, the solution of

$$
\frac{\partial \lambda}{\partial t}=\eta J\left(\frac{S}{p-2 \lambda}, \lambda\right)-\left(k_{d}+d\right) \lambda+\frac{a W}{X_{\infty}}
$$

is calculated using the trapezoidal method, which is equivalent to UCS2 in the absence of convection [20], i.e. it is consistent with the numerical scheme that we use for the PDE system. $S$ and $W$ are calculated here using the boundary conditions for substrate concentration and suspended bacteria concentration along with (2.24).

In the following simulations, we investigate the solution to the system while considering only Monod growth kinetics for both the flux into the biofilm and growth rate of suspended bacteria.

\subsubsection{Simulation without suspended bacteria}

In the first simulation, the effect of suspended bacteria is neglected (i.e. $\mu_{u}=0.0\left[\mathrm{~d}^{-1}\right]$ and $\left.a=0.0\left[\mathrm{~d}^{-1}\right]\right)$ to provide a base case for comparison of our model. This base case is the model described in [1]. The simulation results are visualized in Figure 2. The substrate concentration depletes quite rapidly in the inflow region of the reactor, which 
Table 2. Boundary conditions and altered model parameter values for various simulations

\begin{tabular}{|c|c|c|c|}
\hline Simulation & Boundary conditions & Parameter values & Results \\
\hline $\begin{array}{l}\text { Illustrative simulation with } \\
\text { suspended bacteria and } \\
\text { attachment not considered }\end{array}$ & $\begin{aligned} C_{0} & =1.0\left[\mathrm{gm}^{-2}\right] \\
U_{0} & =0.0\left[\mathrm{gm}^{-2}\right] \\
\lambda_{0} & =0.0025[-]\end{aligned}$ & $\begin{array}{c}\mu_{0}=0.0\left[\mathrm{~d}^{-1}\right] \\
a=0.0\left[\mathrm{~d}^{-1}\right]\end{array}$ & $\begin{array}{l}\text { Section } 3.1 .1 \\
\text { Figure } 2\end{array}$ \\
\hline $\begin{array}{l}\text { Illustrative simulation with } \\
\text { suspended bacteria and } \\
\text { attachment considered }\end{array}$ & $\begin{aligned} C_{0} & =1.0\left[\mathrm{gm}^{-2}\right] \\
U_{0} & =0.0\left[\mathrm{gm}^{-2}\right] \\
\lambda_{0} & =0.0025[-]\end{aligned}$ & $a=0.3\left[\mathrm{~d}^{-1}\right]$ & $\begin{array}{c}\text { Section } 3.1 .2 \\
\text { Figure } 3\end{array}$ \\
\hline $\begin{array}{l}\text { Comparison between base } \\
\text { model and new model }\end{array}$ & $\begin{array}{c}C_{0}=1.0\left[\mathrm{gm}^{-2}\right] \\
U_{0}=10.0\left[\mathrm{gm}^{-2}\right] \\
\lambda_{0}=0.0025[-]\end{array}$ & $\begin{array}{l}a=0.3\left[\mathrm{~d}^{-1}\right] \\
L=0.05[\mathrm{~m}]\end{array}$ & $\begin{array}{l}\text { Section } 3.2 \\
\text { Figure } 4\end{array}$ \\
\hline $\begin{array}{l}\text { Effects of suspended } \\
\text { bacteria and attachment }\end{array}$ & $\begin{aligned} C_{0} & =1.0\left[\mathrm{gm}^{-2}\right] \\
U_{0} & =0.0\left[\mathrm{gm}^{-2}\right] \\
\lambda_{0} & =0.0025[-]\end{aligned}$ & $\begin{array}{c}a=\text { varied }\left[\mathrm{d}^{-1}\right] \\
L=0.05[\mathrm{~m}]\end{array}$ & $\begin{array}{l}\text { Section } 3.3 \\
\text { Figure } 5\end{array}$ \\
\hline $\begin{array}{l}\text { Biofilm formation in } \\
\text { clean bed reactors }\end{array}$ & $\begin{array}{c}C_{0}=1.0\left[\mathrm{gm}^{-2}\right] \\
U_{0}=10.0\left[\mathrm{gm}^{-2}\right] \\
\lambda_{0}=0.0[-]\end{array}$ & $\begin{array}{c}a=\text { varied }\left[\mathrm{d}^{-1}\right] \\
L=0.05[\mathrm{~m}]\end{array}$ & $\begin{array}{l}\text { Section } 3.4 \\
\text { Figure } 6\end{array}$ \\
\hline Variable flow rate & $\begin{aligned} C_{0} & =1.0\left[\mathrm{gm}^{-2}\right] \\
U_{0} & =0.0\left[\mathrm{gm}^{-2}\right] \\
\lambda_{0} & =0.0025[-]\end{aligned}$ & $\begin{array}{c}a=\text { varied }\left[\mathrm{d}^{-1}\right] \\
L=0.05[\mathrm{~m}] \\
Q=\text { varied }\left[\mathrm{gm}^{-1}\right]\end{array}$ & $\begin{array}{l}\text { Appendix B.1 } \\
\text { Figure B } 1\end{array}$ \\
\hline $\begin{array}{l}\text { Inclusion of suspended } \\
\text { bacteria death }\end{array}$ & $\begin{array}{c}C_{0}=1.0\left[\mathrm{gm}^{-2}\right] \\
U_{0}=00.0\left[\mathrm{gm}^{-2}\right] \\
\lambda_{0}=0.0025[-]\end{array}$ & $\begin{array}{c}a=\text { varied }\left[\mathrm{d}^{-1}\right] \\
L=0.05[\mathrm{~m}] \\
k_{d u}=0.4\left[\mathrm{~d}^{-1}\right]\end{array}$ & $\begin{array}{l}\text { Appendix B.2 } \\
\text { Figure B } 2\end{array}$ \\
\hline
\end{tabular}

is consistent with the Iwasaki model for sand filters described in [25]. In the downstream region, the concentration levels off. The concentration level decreases as time increases. The concentration profile is reflected in the biofilm thickness.

The biofilm grows near the inlet and stratifies further down the flow channel. In the downstream region, the biofilm shows net loss, being thinner than at initial time. This is a consequence of substrate depletion. Low substrate concentrations in the aqueous phase lead to small substrate fluxes into the biofilm, compared to cell death and biofilm loss rates, whereas in the inflow region the strong detachment losses into the aqueous phase are compensated by growth, they dominate in the downstream region.

\subsubsection{Simulation with suspended bacteria}

In the second simulation, the suspended bacteria compartment is introduced with a growth rate of $\mu_{u}=6.0\left[\mathrm{~d}^{-1}\right]$ and the effects that suspended bacteria have on the solution to (2.25) are examined. In this simulation, there are no suspended bacteria added to the channel at 
(a)

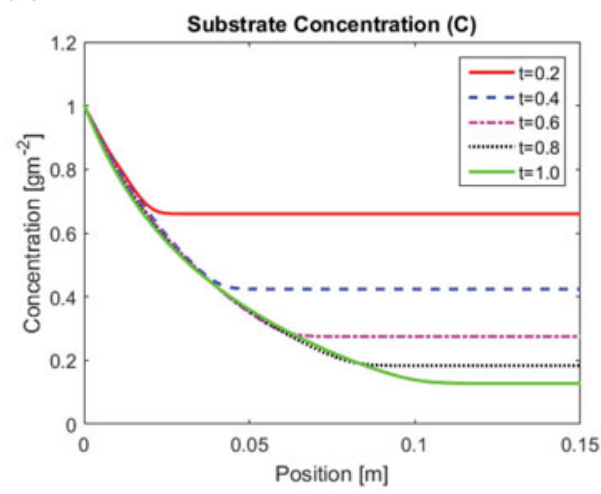

(c)

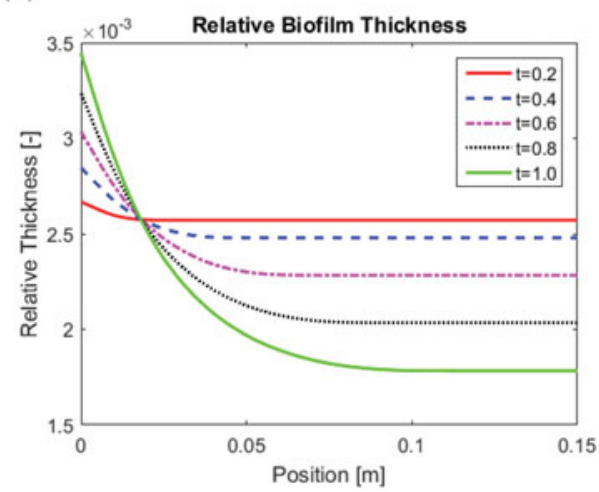

(b)

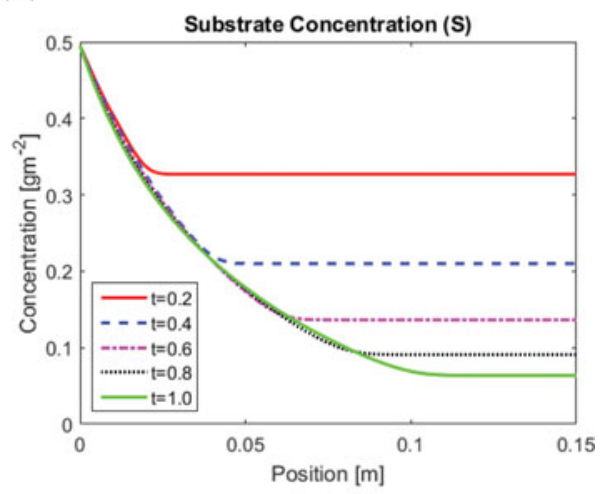

(d)

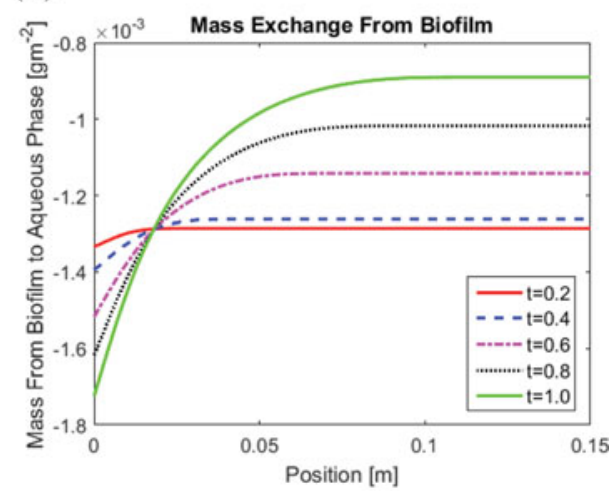

FIGURE 2. Simulation of (2.25) with non-linear flux inside the biofilm layer and without suspended bacteria. Parameter values reported in Table 1. Concentration for original substrate $(C)$ and transformed variable $(S)$, as well as relative biofilm thickness are captured throughout the reactor at different times. Inflow concentration of substrate and suspended bacteria are $C_{0}=1.0\left[\mathrm{gm}^{-2}\right.$ ] and $U_{0}=0.0\left[\mathrm{gm}^{-2}\right]$, respectively.

the inlet (i.e. $U_{0}=0.0\left[\mathrm{gm}^{-2}\right]$ ), i.e. suspended bacteria concentration stems from biomass becoming detached from the biofilm. In this simulation, suspended bacteria can attach to the substratum or the existing biofilm. Suspended bacteria also contribute to substrate degradation for growth before being washed out of the reactor. In this simulation, we therefore give up the assumption of [1] that detached biomass is immediately removed.

The results are visualized in Figure 3. Again, the substrate concentration is rapidly depleted. However, with the introduction of substrate consuming suspended bacteria, this depletion is faster than in the previous case (cf. Figure 2). Growth of suspended biomass due to substrate consumption amplifies this effect. Suspended bacteria and attachment also plays a role in determining biofilm thickness. In Figure 3(e), the relative biofilm thickness near the end of the flow channel at a given time is larger than the relative biofilm thickness at the same location when the suspended bacteria is not present (cf. Figure 2(c)). Although suspended bacteria consume substrate, resulting in less substrate further down the flow channel, there is still a net increase in biofilm thickness due to attachment. 
(a)

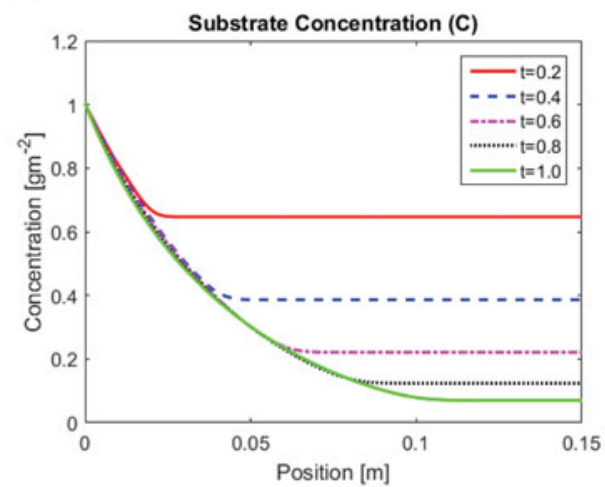

(c)

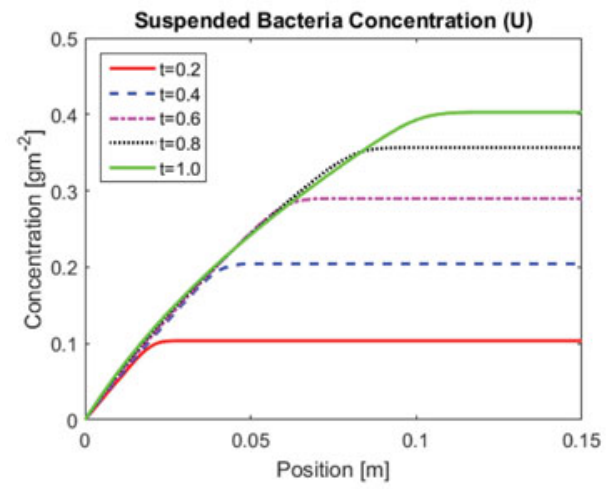

(e)

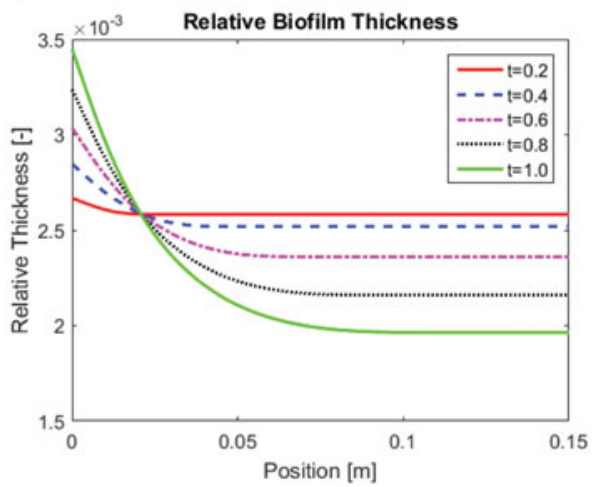

(b)

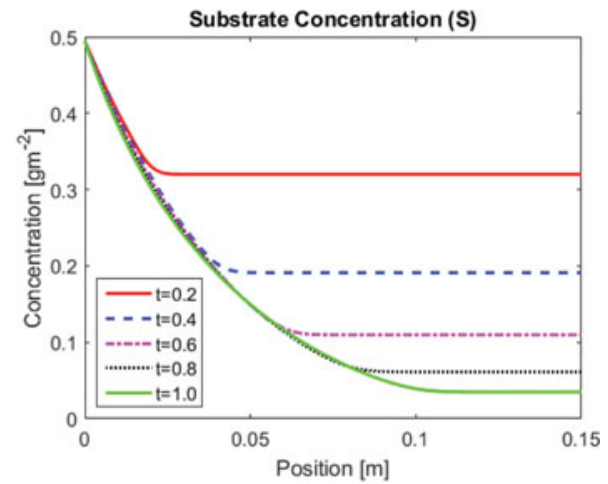

(d)

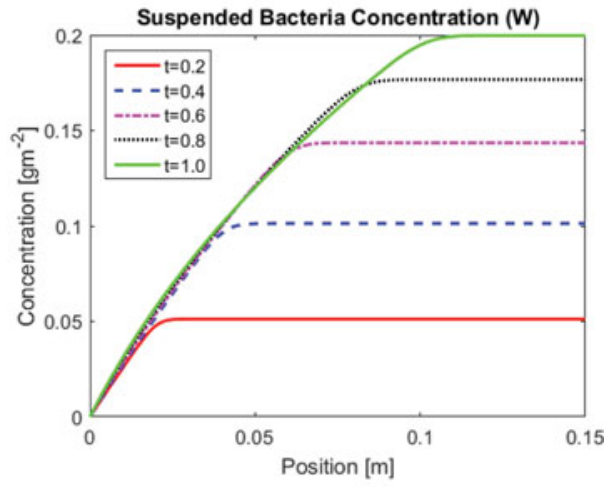

(f)

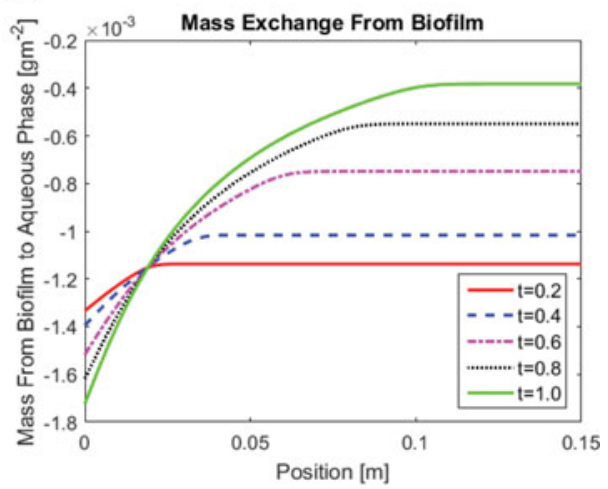

FIGURE 3. Simulation of (2.25) with non-linear flux inside the biofilm layer and suspended bacteria. Parameter values reported in Table 1. Concentration for original substrate $(C)$ and transformed variable $(S)$, original suspended bacteria concentration $(U)$ and transformed variable $(W)$, and relative biofilm thickness are captured throughout the reactor at different times. Inflow concentration of substrate and suspended bacteria are $C_{0}=1.0\left[\mathrm{gm}^{-2}\right]$ and $U_{0}=0.0\left[\mathrm{gm}^{-2}\right]$ respectively. 
In fact, Figure 3(c) illustrates that the suspended bacteria concentration begins to increase over time leading to higher attachment. We also see that as the substrate nears depletion $(t=1.0[\mathrm{~d}])$ there is less net mass exchange from the biofilm into the aqueous phase. Since suspended bacteria are still able to attach after substrate is depleted, a decreased net loss results (cf. Figure 3(f)).

Overall, these two simulations illustrate that suspended bacteria and attachment affects the solution of (2.25). Since suspended bacteria also consume substrate, there is less substrate available for biomass production in the biofilm. However, attachment can still allow the biofilm thickness to increase. The role of suspended bacteria is essential to the proper description of a porous medium biofilm reactor and must therefore not be neglected.

\subsection{Role of suspended biomass in substrate removal}

Going forward we refer to the model without suspended biomass and attachment as the base model, in reference to [1], and the model in which these factors are considered as the new model, as it has been introduced here first.

In the first comparative simulation of both models, we investigate how the suspended bacteria and the attachment rate affect the substrate concentration at outflow, the substrate removal rate, the total biomass in the reactor, and the total reactor biofilm biomass. Here, the reactor length is fixed to be $L=0.05[\mathrm{~m}]$ and the attachment rate is $a=0.3\left[\mathrm{~d}^{-1}\right]$. In these simulations the inflow conditions are set to $C_{0}=1.0\left[\mathrm{gm}^{-2}\right], U_{0}=10.0\left[\mathrm{gm}^{-2}\right]$, and there is an assumed biofilm with relative thickness $\lambda_{0}=0.0025[-]$. Simulation results are reported in Figure 4.

In Figure 4(c), the maximum amount of biomass occurs much sooner in the new model, and at much higher levels, whereas, as seen in Figure 4(a), the substrate concentration profile is much lower in the new model than the base case. The higher biomass in the reactor in the new model is reflected by a thicker biofilm, cf. Figure 4(d), although most of the biomass in the new model is in the suspended phase. Since suspended biomass in the aqueous phase experiences higher substrate concentrations than the biofilm, it can grow faster and is more efficient in removing substrate from the reactor despite being washed out. In this simulation, suspended biomass was added to the system on inflow. It did have no effect on the base model, but in the new model, it dominated the reactor performance, with biofilm contributions being nearly negligible. This simulation highlights the importance of the inclusion of suspended bacteria to substrate removal.

\subsection{The role of (re-)attachment of suspended bacteria}

We now investigate the effect of attachment on the system described by (2.25) by varying the attachment rate. The reactor length is set to $L=0.05[\mathrm{~m}]$ and the inflow concentration for substrate and suspended bacteria are set to $C_{0}=1.0\left[\mathrm{gm}^{-2}\right]$ and $U_{0}=0.0\left[\mathrm{gm}^{-2}\right]$, respectively. Thus, unlike the previous section, we consider now again the case with no suspended biomass on inflow. The reactor is assumed to have an established biofilm on the porous fabric with a relative biofilm thickness of $\lambda_{0}=0.0025$ [-]. The value of the attachment rate $a$ is increased from $0.0\left[\mathrm{~d}^{-1}\right]$ to $1.0\left[\mathrm{~d}^{-1}\right]$ by increments of 0.2 . 
(a)

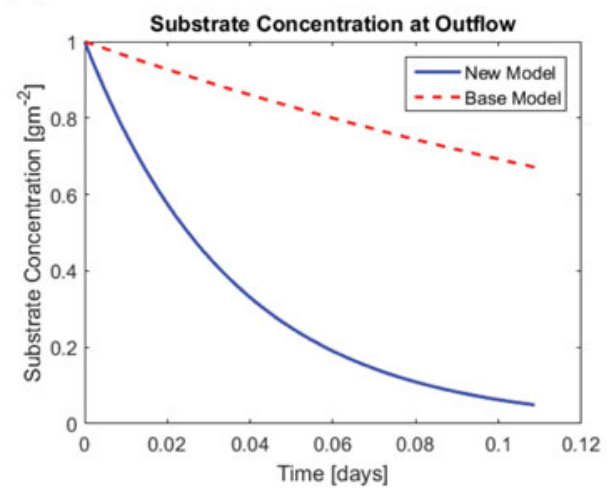

(c)

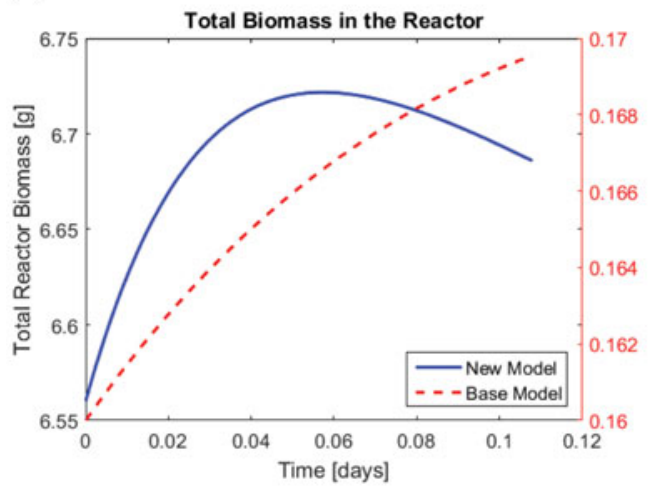

(b)

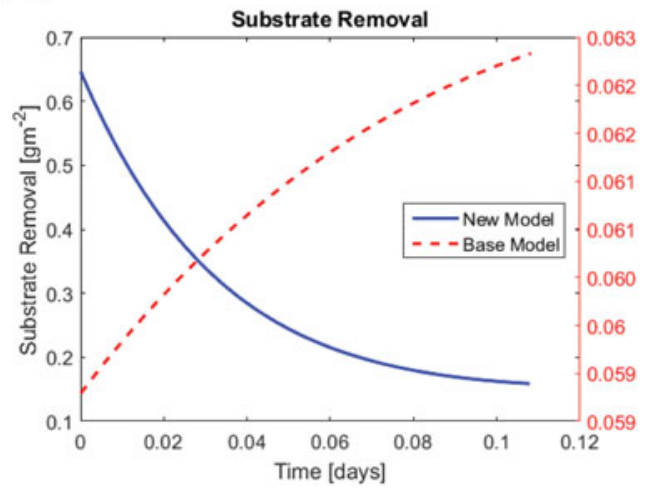

(d)

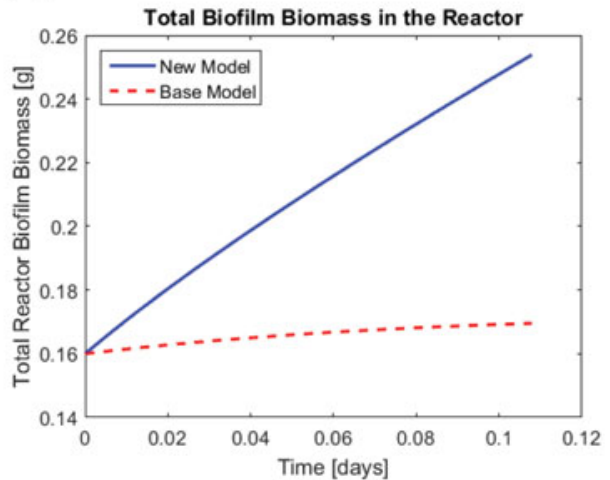

Figure 4. Comparison of the new model and the base case in a $5 \mathrm{~cm}$ reactor. Inflow concentration of substrate and suspended bacteria are $C_{0}=1.0\left[\mathrm{gm}^{-2}\right], \mathrm{U}_{0}=10.0\left[\mathrm{gm}^{-2}\right]$, respectively. Here, the reactor is assumed to have an established biofilm with a relative thickness of $\lambda_{0}=0.0025$ [-].

In these simulations, when $a=0.0\left[\mathrm{~d}^{-1}\right]$ detached biomass does not reattach to the biofilm; however, detached biomass consumes substrate in the aqueous phase, which is fundamentally different than the base model where detached biomass becomes inactive. All other parameter values are listed in Table 1.

Results are reported in Figure 5. An increase in the attachment rate corresponds to a decrease in suspended biomass and an increase in biofilm biomass. Figure 5(b) illustrates a significant difference in suspended biomass of $21 \%$ between the attachment rates of $a=0.0\left[\mathrm{~d}^{-1}\right]$ and $a=1.0\left[\mathrm{~d}^{-1}\right]$. We also see from Figure 5(c) that the total biomass in the reactor increases as the attachment rate increases. For the first 0.5 days the value of the attachment rate does not play a significant role in determining the total biomass in the reactor. However, after 0.5 days, we see that in simulations with higher attachment rates, both the amount of total biomass and biofilm biomass are higher. For biofilm biomass, there is approximately a $17 \%$ difference between the cases of no attachment $a=0.0\left[\mathrm{~d}^{-1}\right]$ and an attachment rate of $a=1.0\left[\mathrm{~d}^{-1}\right]$, which suggests attachment has a significant effect on biomass retained in the biofilm. The increase in biofilm biomass is consistent with a decrease in suspended biomass for corresponding attachment rates. 
(a)

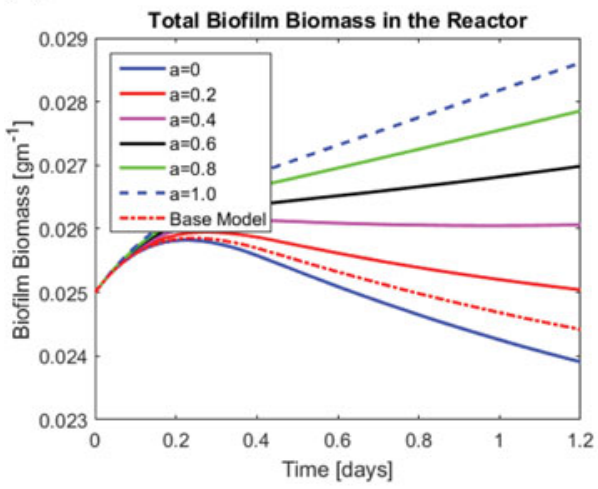

(c)

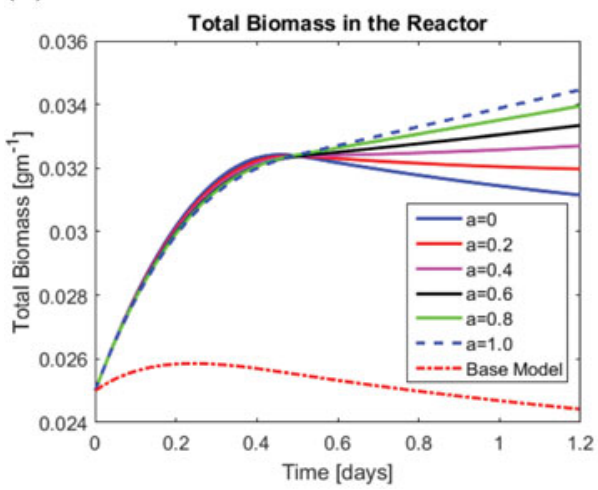

(b)

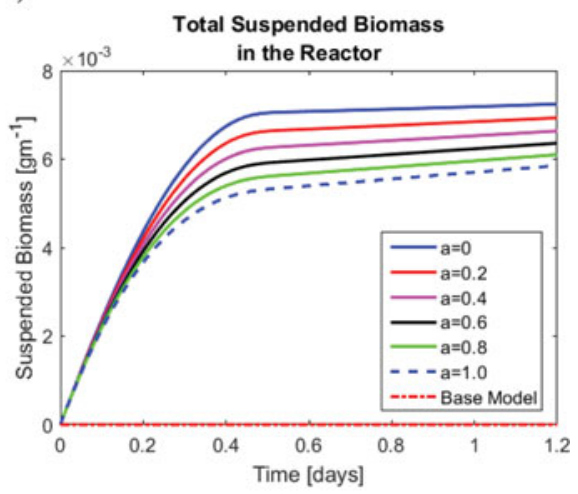

(d)

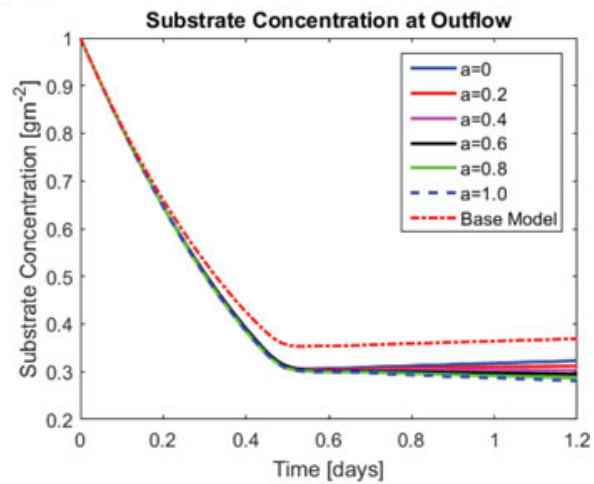

FIGURE 5. The effect of non-linear flux and variable attachment rates on (a) biofilm biomass, (b) suspended biomass, (c) total biomass, and (d) substrate concentration in a $5 \mathrm{~cm}$ reactor. Inflow concentration of substrate and suspended bacteria are $\mathrm{C}_{0}=1.0\left[\mathrm{gm}^{-2}\right], \mathrm{U}_{0}=0.0\left[\mathrm{gm}^{-2}\right]$, respectively. Here, the reactor was assumed to have an established biofilm with a relative thickness of $\lambda_{0}=$ 0.0025 [-]. Parameter values reported in Table 1.

Since the amount of biomass in the biofilm is much larger than the total suspended biomass (approx. four times larger), the increase in biofilm biomass is larger than the loss in suspended biomass yielding a net gain in total biomass. It is important to note that suspended biomass is less than biofilm biomass and biofilm biomass is much less than the void fraction, i.e. $U<\lambda \ll p / 2$. This provides also an a posteriori justification for the assumption that the volume occupied by suspended bacteria can be neglected.

Figure 5(d) shows that the substrate concentration in the new model is lower than in the base model without suspended growth and reattachment. We see a $13 \%$ difference in substrate concentration between the cases where suspended bacteria is included with an attachment rate of $a=0.0\left[\mathrm{~d}^{-1}\right]$, and the case where it is neglected, indicating that neglecting these effects will underestimate reactor performance. In the initial phase, this seems to be dominated by suspended growth alone, as all attachment rates yield similar results. After the first 0.5 days, the substrate concentration evolves slightly differently for different attachment rates, in the sense that higher attachment leads to slightly 
lower substrate concentrations. Additionally, after 1.2 days, we see a $14 \%$ difference in substrate concentration between no attachment $a=0.0\left[\mathrm{~d}^{-1}\right]$ and an attachment rate of $a=1.0\left[\mathrm{~d}^{-1}\right]$, suggesting that the attachment rate does significantly contribute to substrate removal. The effect of varying the attachment rate is more pronounced for the biomass in the reactor (cf. Figure 5(c)).

Again, we stress that in Figure 5 the case when the attachment rate is $a=0.0\left[\mathrm{~d}^{-1}\right]$ is a fundamentally different case than the base case. When the attachment rate is zero, we still have suspended bacteria playing a role in the system, i.e. suspended bacteria are not immediately washed out of the reactor. Thus, unlike the base case, suspended bacteria still consume substrate to promote growth in the aqueous phase. As seen in Figure 5(d) a major effect of substrate depletion is given by the presence of suspended bacteria, which is consistent with the findings in [22]. Furthermore, we find that the attachment process also contributes significantly to substrate degradation.

We also considered the case when the mesoscopic detachment expression vanishes in the continuous limit and the macroscopic model (2.21) simplifies to $d=0.0\left[\mathrm{~d}^{-1}\right]$. In the absence of suspended bacteria on inflow, this simulation configuration simplifies our model and becomes the model described in [1] while neglecting detachment. With no suspended bacteria in the reactor, attachment would have no effect and the biofilm would grow strictly by consuming substrate via the concentration gradient. Adding suspended bacteria at inflow would mimic the case from Figure 5 when $a>d$, resulting in an increased loss to suspended biomass and an increase in the biofilm thickness.

\subsection{Applications to clean bed simulations}

Finally, we investigate whether or not a biofilm can form inside an empty reactor via passing growth substrate and suspended bacteria through the reactor. We call an empty porous medium reactor having no established biofilm on its surface a clean bed.

For this simulation, we rescale our reactor to a length of $\mathrm{L}=0.05[\mathrm{~m}]$ and assume the reactor has a clean bed. Growth substrate and suspended bacteria are added at the inlet with concentrations of $C_{0}=1.0\left[\mathrm{gm}^{-2}\right]$ and $U_{0}=10.0\left[\mathrm{gm}^{-2}\right]$, respectively. The attachment rate is increased from $0.2\left[\mathrm{~d}^{-1}\right]$ to $0.8\left[\mathrm{~d}^{-1}\right]$ by increments of 0.2 to determine if higher attachment rates affect biofilm formation. The results are reported in Figure 6.

Figure 6(a) shows that it is possible to promote biofilm growth on a clean bed. At time $t=1.0\left[\mathrm{~d}^{-1}\right]$ even for a low attachment rate of $a=0.2\left[\mathrm{~d}^{-1}\right]$, a biofilm with a relative thickness of $\lambda=6.1 \times 10^{-3}[-]$, which occupies approximately $2.4 \%$ of the void fraction, is established. From Figures 6(c) and (d), we see that as the attachment rate is increased the biofilm thickness also increased, with the thickest values occurring at the inlet. As time progresses, the biofilm thickness continues to grow linearly, despite substrate becoming extremely limited. This limitation is due to the abundance of suspended bacteria that is constantly being added to the system at the inlet. The suspended bacteria is able to attach to the biofilm, which causes the biofilm to grow linearly once the substrate becomes depleted. For the same reason, when the attachment rate is increased, the biofilm thickness at the end of the flow channel is also increased.

In Figure 6(e), we plot the effect of attachment on substrate concentration at a specific location in the reactor $(0.007[\mathrm{~m}])$. Despite there being a relatively high concentration 
(a)

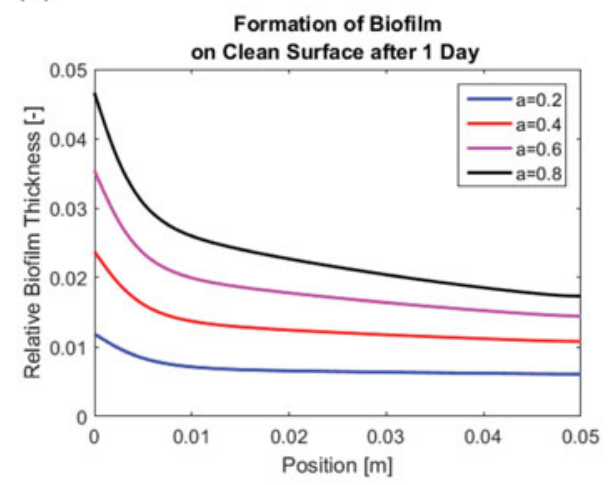

(c)

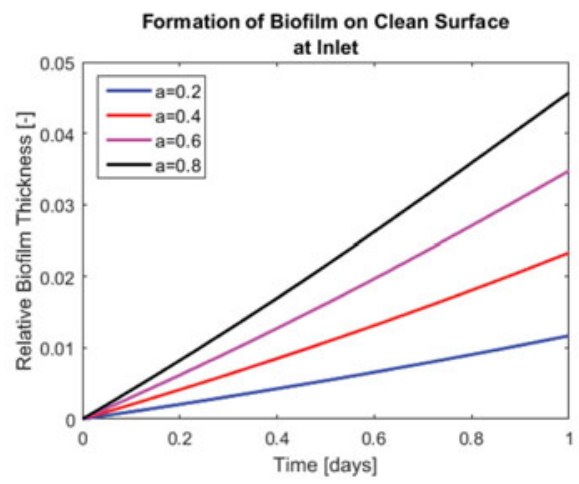

(e)

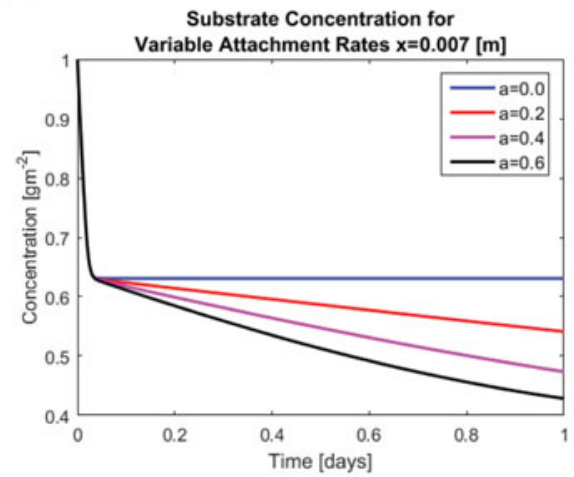

(b)

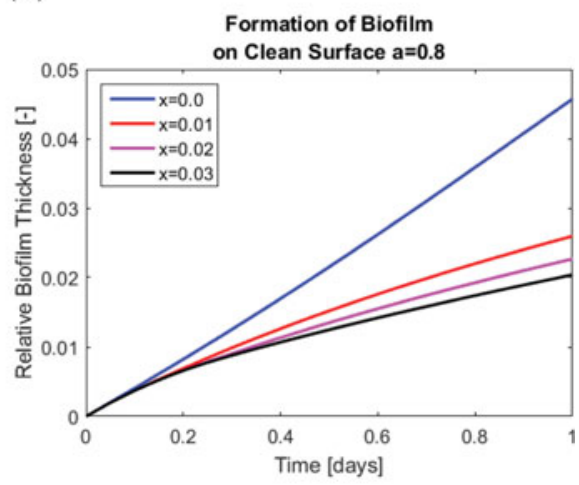

(d)

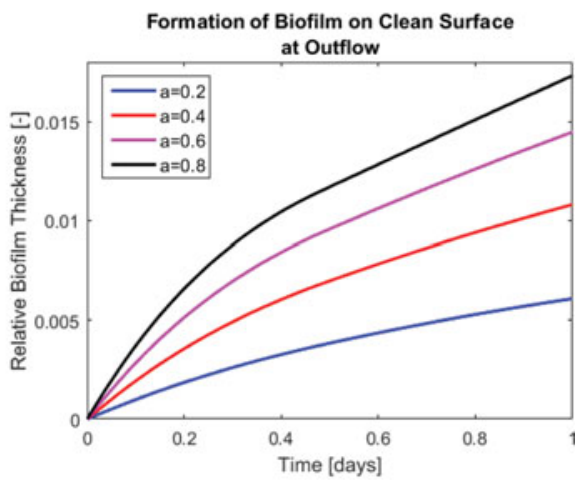

(f)

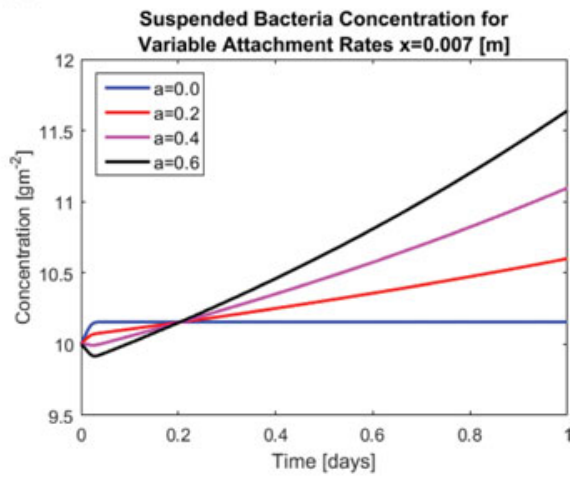

FIGURE 6. Simulation of (2.25) on a clean bed (i.e. no established biofilm in the reactor). Inflow concentration of substrate and suspended bacteria are $C_{0}=1.0\left[\mathrm{gm}^{-2}\right]$ and $U_{0}=10.0\left[\mathrm{gm}^{-2}\right]$, respectively. Multiple attachment rates are used to determine the effect on substrate concentration and relative biofilm thickness. Parameter values reported in Table 1.

of suspended bacteria in the reactor, we see that substrate is consumed more rapidly at higher attachment rates. An important feature to note is that the lowest consumption of substrate occurred when there was zero attachment $\left(a=0.0\left[\mathrm{~d}^{-1}\right]\right)$. This shows that although suspended bacteria is capable of removing substrate from the reactor, 
a combination of suspended bacteria and attached bacteria is more efficient [22], as residence times increase with attachment.

Figure 6(f) illustrates the response of suspended bacteria concentration to increased attachment rates at a specific location in the reactor $(0.007[\mathrm{~m}])$. We see for higher attachment rates, suspended bacteria concentration initially decreases. This initial decrease corresponds to the increase in biofilm thickness. In earlier stages of the simulation, the biofilm is not very well established, i.e. the relative thickness is still quite small. In this earlier stage, the increase in suspended bacteria is dominated by suspended growth rather than detachment due to the fact that detachment is proportional to biofilm thickness. As time increases and substrate concentration decreases (cf. Figure 6(e)) suspended bacteria growth becomes limited. Simultaneously, biofilm thickness increases (cf. Figures 6(c) and (d)) resulting in more detached biomass. Thus, the primary contribution to suspended bacteria changes from bacterial growth to detachment of biofilm biomass.

These simulations provide insight into the formation of a biofilm on a clean surface so long as there is sufficient bacteria in the bulk liquid and the attachment rate is large enough.

\section{Discussion}

The objectives of this study were to derive a multiscale model for biofilms in porous media and investigate the effects of suspended bacteria and attachment on the macroscopic reactor.

By comparing two models, one without and one with suspended bacteria and attachment, we observed a more rapid depletion of substrate concentration. This result is consistent with other biofilm reactor models, such as the CSTR models in [17,22]. Suspended biomass is exposed to higher substrate concentrations and therefore has a growth advantage over biofilm biomass, leading to faster overall growth. Attachment in the model prevents some of the suspended biomass from being washed out; however, the amount of suspended bacteria present in the reactor is affected by the flow rate. Attachment increases bacterial residence time and can lead to thicker biofilms due to deposition. Thus, biofilm in downstream regions can increase in thickness even if substrate concentrations are unfavourable for growth in its inner layers.

By increasing the attachment rate, we initially saw no effect on substrate concentration. As time progressed, we observed variations in the substrate concentration due to the varied attachment rate. These variations suggest that the mesoscopic attachment expression, under certain conditions, does significantly affect reactor performance. An increased attachment rate resulted in less suspended biomass in the reactor and a thicker biofilm layer.

Additionally, a decrease in substrate concentration strictly due to the inclusion of suspended bacteria was found as compared to the model that had suspended bacteria removed. As in [22], for a CSTR, we observed that the amount of suspended biomass in the system was minor compared to biofilm biomass; however, we saw that suspended bacteria was relatively more efficient at substrate removal than biofilm biomass. This is due to the substrate concentration gradient found inside the biofilm. Since inner layers of the biofilm are subject to small concentrations of substrate, their contribution to substrate 
removal is minimal. Decreasing the biofilm thickness, e.g. as a consequence of detachment, does not necessarily reduce the thickness of the active layer in the biofilm. An assumption of this model was to neglect the effect of natural cell death for biomass in the aqueous phase. An a posteriori investigation into the effect of suspended bacteria death, included in Appendix B.2, determined that although suspended bacteria death largely effects the amount of suspended biomass in the reactor, the effect on substrate removal is dampened and the overall qualitative behaviour remains unchanged. Although suspended bacteria is more efficient at substrate removal, neglecting wall attached biofilms would over-predict washout, and thus underestimate reactor performance. This result is in accordance with the CSTR model of [22].

With suspended bacteria leading to an increased removal of substrate, a re-investigation into the effects of mesoscopic detachment expressions on the macroscopic reactor may be appropriate. Simulations in [1] found that the detachment rate coefficient affected the amount of biomass detached from the biofilm. Moreover, the slight increase in detachment was found to have only a minor effect on substrate removal. However, the simulations carried out in [1] neglected suspended bacteria. An increase in biofilm detachment would lead to an increase in suspended bacteria and possibly affect substrate removal.

When investigating the effect of suspended bacteria and attachment on a clean reactor, we observed biofilm formation along the reactor walls. Even for small attachment values $\left(a=0.2\left[\mathrm{~d}^{-1}\right]\right)$ formation of a biofilm that occupied $2.4 \%$ of the void fraction was possible with the thickest portion of the biofilm occurring at the inlet. We also observed the effectiveness of only suspended bacteria on substrate consumption (clean reactor with $\left.a=0.0\left[\mathrm{~d}^{-1}\right]\right)$. We found that suspended bacteria was relatively efficient at removing substrate; however, suspended bacteria along with biofilm biomass was much more effective.

Like most models, simplifying assumptions made limit the models ability to accurately model the described physical system. One limitation of our model is the assumption that the reactor maintains a constant flow. Underlying here is the implicit assumption that the biofilm layer is infinitesimally thin and therefore has no effect on fluid flow. In the above simulations, the relative biofilm thickness never approaches the clogging state $(\lambda=p / 2)$; thus, hydrodynamic forces are minimal and the model does not break down. An investigation into the effect of variable specific flow rates was conducted and the results are reported in Appendix B.1. For larger flow rates, suspended biomass is washed out of the reactor before they are able attach or contribute to substrate degradation. A consequence of this result is that the substrate concentration is larger throughout the reactor, which also allows for a larger biofilm thickness due to substrate availability. We find that although quantitative results are different for variable specific flow rates, qualitatively the reactor behaves in the same manner. Alternatively, to overcome this limitation, the model can be adapted to consider a pressure driven flow field, which may also have implications on both the attachment and detachment rate expressions. By adapting the model to a pressure driven flow field, shear dependent attachment/detachment expressions may be considered. A second limitation of our model results from the assumption that the biofilm covers the substratum in a homogeneous layer. In reality this is not the case. Biofilms are very complex and their structure/development may depend on a variety of different factors such as substrate concentration availability and hydrodynamic conditions. 


\section{Conclusion}

In this paper, we studied a mathematical model for biofilm formation inside a porous medium reactor. By including suspended bacteria, we were able to extend the model outlined in [1] and investigate the effect that attachment and suspended bacteria have on reactor performance.

Through numerical simulation, we illustrated that the inclusion of suspended bacteria is essential for the proper description of the porous medium reactor. Suspended biomass was found to be relatively more efficient at substrate removal than biofilm biomass. This can be explained by the fact that substrate is more readily available to suspended biomass than biofilm biomass, where inner layers only have limited access to substrate through a concentration gradient.

While the specific choice of mesoscopic attachment rate expression may not largely affect the macroscopic model quantitatively under all conditions, its inclusion is essential in some cases. For example, it allows for biofilm formation on a clean bed. The inclusion of suspended bacteria has major implications on reactor performance and biofilm thickness. Suspended bacteria greatly contribute to the depletion of substrate, causing the reactor to enter a substrate limiting regime sooner. Although suspended biomass is more efficient in removing substrate, it is prone to washout. Attachment increases residence time and mechanical stability of the reactor operation.

This suggests that models of bacterial growth in porous media should include both biofilm and suspended biomass, as well as detachment and attachment processes.

\section{References}

[1] Abbas, F. \& Eberl, H. (2013) Investigation of the role of mesoscale detachment rate expressions in a macroscale model of a porous medium biofilm reactor. Int. J. Biomath. Biostat. 2(1), 123-143.

[2] Abbas, F. \& Eberl, H. J. (2011) Analytical substrate flux approximation for the monod boundary value problem. Appl. Math. Comp. 218(4), 1484-1494.

[3] Abbas, F., Sudarsan, R. \& Eberl, H. J. (2012) Longtime behavior of one-dimensional biofilm models with shear dependent detachment rates. Math. Biosci. Eng. 9(2), 215-239.

[4] BallyK, M. M., Jones, D. A. \& SMith, H. L. (2001) Microbial competition in reactors with wall attachment: A mathematical comparison of chemostat and plug flow models. Microb. Ecol. 41, 210-221.

[5] Burden, R. L. \& Faires, J. D. (2010) Numerical Analysis, Brooks/Cole Publishing, Boston, MA.

[6] Carl, S. \& Heikkilä, S. (2000) Nonlinear Differential Equations in Ordered Spaces. Chapman \& Hall, Boca Raton, FL, USA.

[7] Carpentier, B. \& Cerf, O. (1993) Biofilms and their consequences, with particular reference to hygiene in the food industry. J. Appl. Bacteriol. 75(6), 499-511.

[8] D’Acunto, B., Frunzo, L., Klapper, I. \& Mattei, M. R. (2015) Modeling multispecies biofilms including new bacterial species invasion. Math Biosci. 259, 20-26.

[9] Davit, Y., Byrne, H., Osborne, J., Pitt-Francis, J., Gavaghan, D. \& Quintard, M. (2013) Hydrodynamic dispersion within porous biofilms. Phys. Rev. E 87, 1-16.

[10] Donlan, R. M. (2002) Biofilms: Microbial life on surfaces. Emerg. Infect. Dis. 82(1), 881-890.

[11] Duddu, R., Chopp, D. L. \& Moran, B. (2009) A two-dimensional continuum model of biofilm growth incorporating fluid flow and shear stress based detachment. Biotechnol. Bioeng. 103(1), 92-104. 
[12] Emerenini, B., Hense, B. A., Kuttler, C. \& Eberl, H. J. (2015) A mathematical model of quorum sensing induced biofilm detachment. PloS ONE 10(7), e0132385.

[13] Epperson, J. F. (2002) An Introduction to Numerical Methods and Analysis. John Wiley \& Sons, New York, NY, USA.

[14] Gaebler, H. J. (2017) Mulit-scale model formulation of a porous medium biofilm reactor and the effects of suspended bacteria, mesoscopic attachment, and growth kinetics on the macroscopic reactor. Master's thesis, University of Guelph, ON, Canada.

[15] Golfier, F., Wood, B. D., Orgogozo, L., Quintard, M. \& Bues, M. (2009) Biofilms in porous media: Development of macroscopic transport equations via volume averaging with closure for local mass equilibrium conditions. Adv. Water Resour. 32(3), 463-485.

[16] Huinink, H. (2016) Fluids in Porous Media: Transport and Phase Changes. Morgan \& Claypool Publishers, San Rafael, CA, USA.

[17] Jones, D., Kojouharov, H. V., Le, D. \& Smith, H. (2003) The Freter model: A simple model of biofilm formation. J. Math. Biol. 47, 137-152.

[18] Kang, Q., Lichtner, P., Viswanathan, H. \& Abdel-Fattah, A. (2010) Pore scale modeling of reactive transport involved in geologic $\mathrm{CO}_{2}$ sequestration. Transp. Porous Media 8(9), 197-213.

[19] Klapper, I. (2012) Productivity and equilibrium in simple biofilm models. Bull. Math. Biol. 74(12), 2917-2934.

[20] Liotta, S. F., Romano, V. \& Russo, G. (2000) Central schemes for balance laws of reaction type. SIAM. J. Numer. Anal. 38(4), 1337-1356.

[21] Logan, J. D. (2000) Transport Modeling in Hydrogeochemical Systems. Springer-Verlag, New York, NY, USA.

[22] MašÍ́, A. \& Eberl, H. J. (2012) Persistence in a single species CSTR model with suspended flocs and wall attached biofilms. Bull. Math. Biol. 75(5), 1001-1026.

[23] Mašić, A. \& EberL, H. J. (2016) A chemostat model with wall attachment: The effect of biofilm detachment rates on predicted reactor performance. In: J. Bélair, I. Frigaard, H. Kunze, R. Makarov, R. Melnik \& R. Spiteri (editors), Mathematical and Computational Approaches in Advancing Modern Science and Engineering, Springer International Publishing, Cham, pp. 267-276.

[24] Morgenroth, E. (2003) Detachment: An often-overlooked phenomenon in biofilm research and modelling. In: S. Wuertz, P. L. Bishop \& P. A. Wilderer (editors), Biofilms in Wastewater Treatment, IWA Publishing, London, England, pp. 246-290.

[25] Nieć, J., Spychala, M. \& Zawadzki, P. (2016) New approach to modelling of sand filter clogging by septic tank effluent. J. Ecol. Eng. 17(2), 97-107.

[26] Picioreanu, C., Van Loosdrecht, M. C. M. \& Heijnen, J. J. (2000) Effect of diffusive and convective substrate transport on biofilm structure formation: A two-dimensional modeling study. Biotechnol. Bioeng. 69(5), 504-515.

[27] Rittmann, B. E. (1982) The effect of shear stress on biofilm loss rate. Biotechnol. Bioeng. 24, 501-506.

[28] Rittmann, B. E. \& McCarty, P. L. (2001) Environmental Biotechnology: Principles and Applications. McGraw-Hill, Boston, MA, USA.

[29] Rittmann, B. E., Stilwell, D. \& Ohashi, A. (2002) The transient-state, multiple-species biofilm model for biofiltration processes. Water Resour. 36, 2342-2356.

[30] Singh, R., Paul, D. \& JaIn, R. K. (2006) Biofilms: Implications in bioremediation. Trends Microbiol. 14(9), 388-397.

[31] Tiwari, S. K. \& Bowers, K. L. (2001) Modeling biofilm growth for porous media applications. Math. Comput. Model. 33, 299-319.

[32] VafaI, K. (2010) Ed. Porous Media: Applications in Biological Systems and Biotechnology, CNC Press, Boca Raton, FL, USA.

[33] Wanner, O., Cunningham, A. B. \& Lundman, R. (1995) Modeling biofilm accumulation and mass transport in a porous medium under high substrate loading. Biotechnol. Bioeng. 47, 703-712. 
[34] Wanner, O., Eberl, H. J., Morgenroth, E., Noguera, D. R., Picioreanu, C., Rittmann, B. E. \& van Loosdrecht, M. (2006) Mathematical Modeling of Biofilms. IWA Publishing, London, England.

[35] Wanner, O. \& Gujer, W. (1986) A multispecies biofilm model. Biotechnol. Bioeng. 28, 314-386.

[36] Wood, B., Radakovich, K. \& Golfier, F. (2007) Effective reaction at a fluid-solid interface: Applications to biotransformation in porous media. Adv. Water Resour. 30, 1630-1674.

\section{Appendix A Discretization of the partial differential equation}

To solve the model given by (2.25), we employ the second-order Uniformly accurate Central Scheme UCS2 for hyperbolic conservation laws that was originally introduced in [20]. Problems of this type take the form

$$
u_{t}+(\hat{f}(u))_{x}=h(u)
$$

Here, $u$ represents the dependent variable, $\hat{f}(u)$ describes the nonlinear fluxes that are obtained by passing to the limit, and $h(u)$ describes all reaction terms, i.e.

$$
u=\left(\begin{array}{c}
S \\
W \\
\lambda
\end{array}\right), \hat{f}(u)=\left(\begin{array}{c}
\frac{S Q}{p-2 \lambda} \\
\frac{W Q}{p-2 \lambda} \\
0
\end{array}\right), h(u)=\left(\begin{array}{c}
-2 J\left(\lambda, \frac{S}{p-2 \lambda}\right)-\frac{1}{Y_{u}} g\left(\frac{S}{p-2 \lambda}\right) W \\
2 X_{\infty} d \lambda-2 a W+g\left(\frac{S}{p-2 \lambda}\right) W \\
\frac{Y_{\lambda}}{X_{\infty}} J\left(\lambda, \frac{S}{p-2 \lambda}\right)-\left(k_{d}+d\right) \lambda+\frac{a W}{X_{\infty}}
\end{array}\right) .
$$

In UCS2, the reaction terms are treated implicitly and the transport terms explicitly. The time step $\Delta t$ is constrained by

$$
\Delta t \leqslant \Delta x \frac{\left(p-2 \max _{j}\left(\lambda_{j}^{n}\right)\right)}{2 Q},
$$

where $j$ is the grid position and $\Delta x$ is the spatial discretization step of a uniform grid.

UCS2 computes two predictor steps for the reaction terms with time steps $\Delta t / 2$ and $\Delta t / 3$ :

$$
\begin{aligned}
& u_{j}^{n+1 / 2}=u_{j}^{n}-\frac{1}{2} \frac{\Delta t}{\Delta x} f_{j}^{\prime}+\frac{\Delta t}{2} h\left(u_{j}^{n+1 / 2}\right), \\
& u_{j}^{n+1 / 3}=u_{j}^{n}-\frac{1}{3} \frac{\Delta t}{\Delta x} f_{j}^{\prime}+\frac{\Delta t}{3} h\left(u_{j}^{n+1 / 3}\right),
\end{aligned}
$$

where $n$ is the previous time step, $j$ is the grid position and $f_{j}^{\prime} / \Delta x$ is a flux limiter approximation of the derivative of $f$. We choose the min-mod function, i.e.

$$
f_{j}^{\prime}=M M\left(\hat{f}_{j+1}-\hat{f}_{j}, \hat{f}_{j}-\hat{f}_{j-1}\right)
$$

with

$$
M M(x, y)= \begin{cases}\operatorname{sgn}(x) \min (|x|,|y|) & \text { if } \operatorname{sgn}(x)=\operatorname{sgn}(y) \\ 0 & \text { otherwise }\end{cases}
$$


The predictor steps are used to compute the solution to the system at the next time step at staggered grid points as a solution of the grid-point local nonlinear algebraic system

$$
\begin{aligned}
u_{j+1 / 2}^{n+1}= & \frac{1}{2}\left(u_{j}^{n}+u_{j+1}^{n}\right)+\frac{1}{8}\left(u_{j}^{\prime}-u_{j+1}^{\prime}\right)-\frac{\Delta t}{\Delta x}\left(\hat{f}\left(u_{j+1}^{n+1 / 2}\right)-\hat{f}\left(u_{j}^{n+1 / 2}\right)\right) \\
& +\Delta t\left(\frac{3}{8} h\left(u_{j}^{n+1 / 3}\right)+\frac{3}{8} h\left(u_{j+1}^{n+1 / 3}\right)+\frac{1}{4} h\left(u_{j+1 / 2}^{n+1}\right)\right),
\end{aligned}
$$

where $u_{j}^{\prime}$ is analogous to (A 3). We use for this Newton's method and compute the Jacobian using a finite difference approximation. To evaluate the function $h$, the substrate fluxes $J$ at the biofilm water interface must be computed. We use a single shooting method for this, based on Newton's method and a time adaptive Runge-Kutta-Felhberg RKF5(4) method. Performance tests and validations for the method for the problem at hand can be found in [14].

\section{Appendix B Additional sensitivity analysis}

In addition to Section 3, an investigation into the effect of variable specific flow rates and the inclusion of suspended bacteria death was conducted to determine if these parameters affected the qualitative results of the system.

\section{B.1 Variable flow rate}

The results from varying the flow rate are reported in Figure B 1. In these simulations, the conditions from Figure 5 (i.e. $5 \mathrm{~cm}$ reactor, variable attachment rates, $C_{0}=1.0\left[\mathrm{gm}^{-2}\right.$, $\left.U_{0}=0.0\left[\mathrm{gm}^{-2}\right]\right)$ are re-simulated for different flow rate values, $Q=0.01, Q=0.025$, $Q=0.05$, and $Q=0.1\left[\mathrm{md}^{-1}\right]$.

Simulations indicate that for smaller flow rate values more suspended bacteria remains in the system. As the flow rate is increased, the wash out rate also increases, resulting in a decrease in suspended bacteria. When the flow rate is less than $Q=0.5$, the attachment rate becomes more significant in determining the suspended bacteria concentration. Figures B 1(a) and (c) also illustrate how the flow rate effects the biomass in the system. We see that there is more biofilm biomass at a higher flow rate. This can be explained by the fact that biofilm processes are not influenced by the flow rate. Additionally, as the flow rate is increases and suspended biomass decreases, the amount of substrate remaining in the system changes (cf. Figure B 1(d)). With less suspended biomass the substrate concentration near the end of the reactor is larger, allowing the biofilm to continue to grow. Similar to the results of Figure 5, we see that the value of the attachment rate has an effect on substrate removal. These results suggest that a variable flow rate will change the quantitative results; however, qualitatively the overall findings remain unchanged.

\section{B.2 Inclusion of suspended bacteria death}

We investigate the effect of suspended bacteria death on the system and determine $a$ posteriori if the assumption to neglect suspended death is valid. For these simulations the reactor length is $5 \mathrm{~cm}$, and it is assumed that there are no suspended bacteria in 
(a)

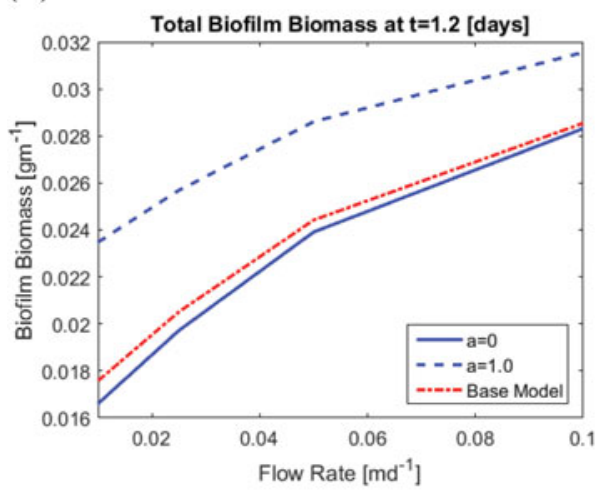

(c)

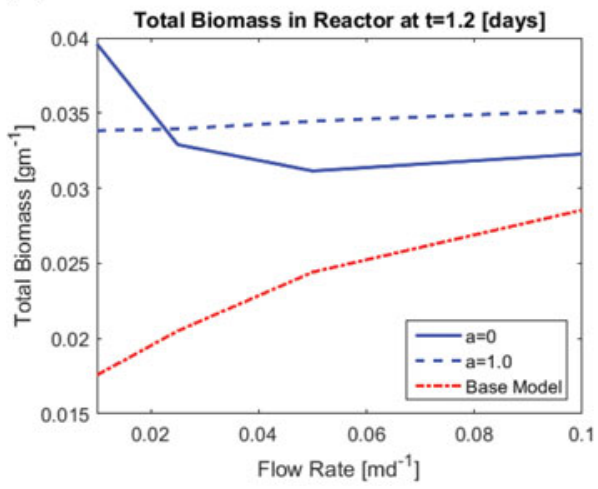

(b)

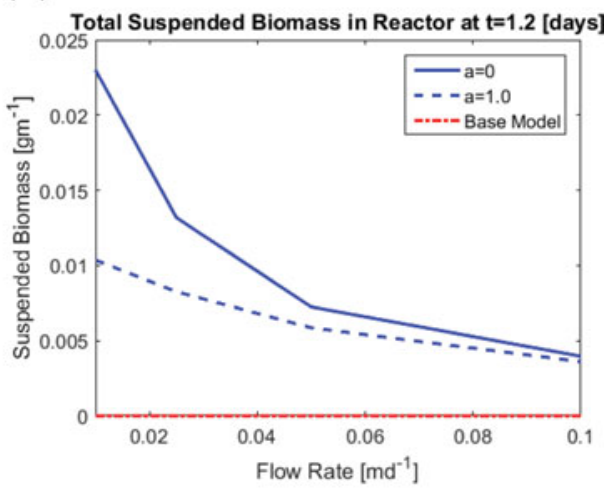

(d)

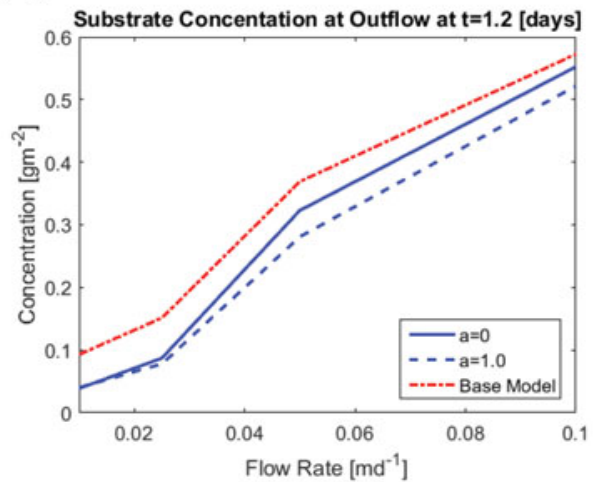

FiguRE B 1. Simulation results for variable flow rate $Q$ in a $5 \mathrm{~cm}$ reactor. Initial substrate concentration $C_{0}=1.0\left[\mathrm{gm}^{-2}\right]$, suspended bacteria concentration, $U_{0}=0.0\left[\mathrm{gm}^{-2}\right]$, and relative biofilm thickness $\lambda_{0}=0.0025[-]$.

the reactor at time $t=0.0$ [d]. The initial concentration of substrate is set to $C_{0}=1.0$ $\left[\mathrm{gm}^{-2}\right]$, and it is assumed there is an established biofilm of relative thickness $\left[\mathrm{gm}^{-2}\right]$. The simulation is run for 1.2 days with different attachment rates. The results are reported in Figure B 2.

Here, we see that the results from the simulations with suspended bacteria death (Figure B 2) are qualitatively the same as the results without suspended bacteria death (Figure 5). Quantitatively, we see a slight reduction in the amount of suspended biomass and biofilm biomass, resulting in a net decrease in total biomass. However, despite the reduction in biomass, the effect on substrate removal is minimal. Results indicate a $17 \%$ difference between the cases of attachment $a=0.0\left[\mathrm{~d}^{-1}\right]$ and $a=1.0\left[\mathrm{~d}^{-1}\right]$ for total biofilm biomass in the system, a $12 \%$ and $13 \%$ difference in substrate concentration between the cases of $a=0.0\left[\mathrm{~d}^{-1}\right]$ and the base model and $a=0.0\left[\mathrm{~d}^{-1}\right]$ and $a=1.0\left[\mathrm{~d}^{-1}\right]$, respectively. Furthermore, we see a $20 \%$ difference in suspended biomass between attachment rates $a=0.0\left[\mathrm{~d}^{-1}\right]$ and $a=1.0\left[\mathrm{~d}^{-1}\right]$. The above differences are consistent with the results from Figure 5. Thus, we are able to confirm a posteriori that our assumption to neglect suspended bacteria death does not have a major effect on the solution to the system. 
(a)

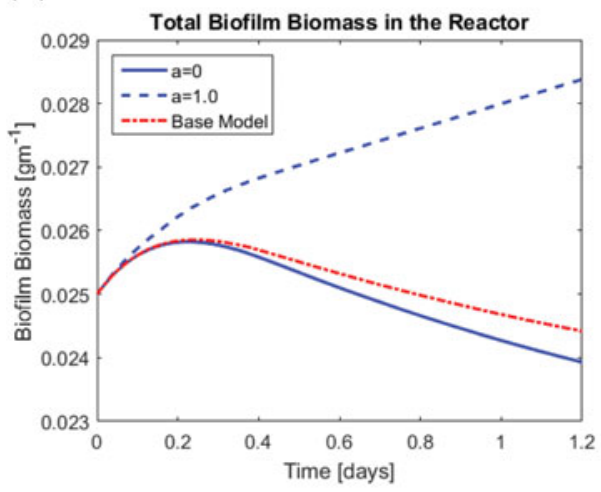

(c)

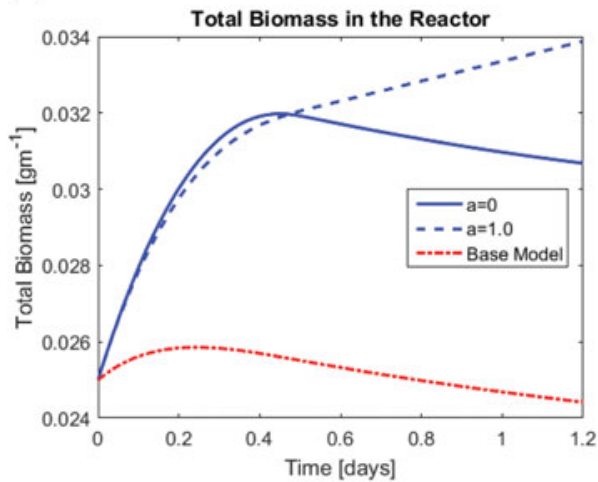

(b)

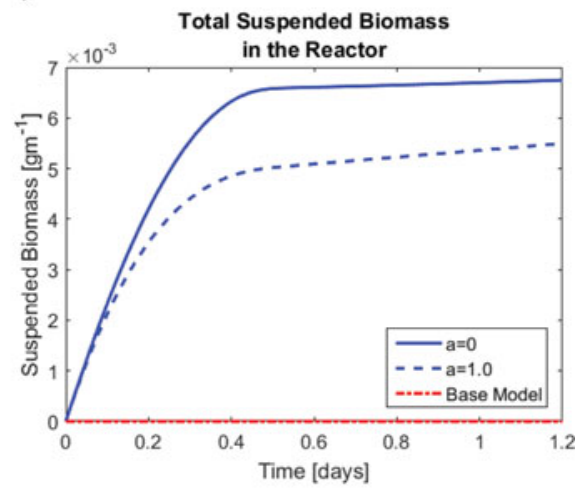

(d)

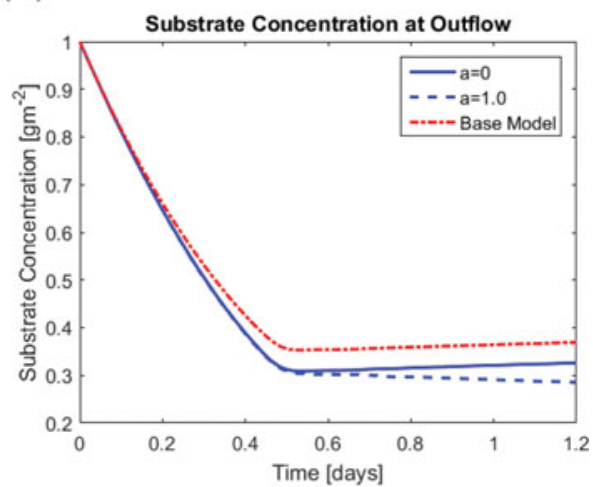

FIGURE B 2. Simulation results of (2.21) with suspended bacteria death and various attachment rates. Initial substrate concentration, $C_{0}=1.0\left[\mathrm{gm}^{-2}\right]$, suspended bacteria concentration, $U_{0}=0.0$ $\left[\mathrm{gm}^{-2}\right]$, and relative biofilm thickness, $\lambda_{0}=0.0025[-]$. 